\title{
Phospholipase D Transduces Force to TREK-1 Channels in a Biological Membrane
}

\author{
E. Nicholas Petersen ${ }^{1,4}$, Manasa Gudheti ${ }^{5}$, Mahmud Arif Pavel ${ }^{1}$, Keith R. Murphy ${ }^{2,3}$, William W. \\ $\mathrm{Ja}^{2,3}$, Erik M. Jorgensen ${ }^{5}$, Scott B. Hansen ${ }^{1^{*}}$ \\ ${ }^{1}$ Departments of Molecular Medicine, ${ }^{2}$ Department of Neuroscience, ${ }^{3}$ Center on Aging, ${ }^{4}$ Scripps Research \\ Skaggs Graduate School of Chemical and Biological Sciences, The Scripps Research Institute, Scripps, \\ Florida 33458, USA \\ ${ }^{5}$ Department of Biology, Howard Hughes Medical Institute, University of Utah, Salt Lake City, UT 84112, \\ USA \\ *Correspondence: shansen@scripps.edu
}

\begin{abstract}
The transduction of force into a biological signal is critical to all living organisms. Recently, disruption of ordered lipids has emerged as an 'atypical' force sensor in biological membranes; however, disruption has yet to link with canonical channel mechanosensation. Here we show that forceinduced disruption and lipid mixing activates TWIKrelated $\mathrm{K}^{+}$channel (TREK-1), and that this activation is dependent on phospholipase D2 (PLD2). PLD2 transduces the force into a chemical signal phosphatidic acid (PA) that is then sensed by TREK- 1 with a latency of $<3$ ms. TREK- 1 then produces a mechanically induced change in membrane potential. Hence, in a biological membrane, we show the ordered lipid is the force sensor, PLD2 is a chemical transducer, and the 'mechanosensitive' ion channel TREK-1 is a downstream effector of mechanical transduction. Confirming this central role for PA singling in force transduction, genetic deletion of PLD decreases mechanosensitivity and pain thresholds in $D$. melanogaster.
\end{abstract}

Keywords: ion channel; lipids; phospholipase D; membrane; mechanosensation; kinetic; lipid rafts;

\section{INTRODUCTION}

All cells must sense force (mechanosensation), not just sensory pain nerves, including those that respond to the heartbeat, skeletal muscle stretch, and epithelial cell adhesion $^{1-3}$, Recent studies show that disruption of ordered proteins and lipids is a non-canonical sensor of mechanical force in biological membranes ${ }^{4,5}$. Force was shown to disassemble and flatten caveolae ${ }^{4}$ and force disrupts ordered lipids and lipid clusters ${ }^{5}$. The ordered phase, sometimes called a lipid raft, is enriched in saturated lipids and palmitoylated proteins ${ }^{6,7}$ and is subject to disruption (see Supplemental Fig. 1a-b). In $\mathrm{C} 2 \mathrm{C} 12$ muscle cells, chemical disruption releases a palmitoylated protein phospholipase $D$ (PLD2) from lipid rafts activating a mechanotransduction pathway (Supplemental Fig. 1c-d). ${ }^{5}$ This mechanical activation of PLD2 is shown to cause muscle cell differentiation ${ }^{5}$, miacropinocytosis ${ }^{8}$, and may act as a force senor upstream of $\mathrm{mTOR}^{9}$. These data point to lipid domains as bona fide sensors of force.

The downstream effector molecules of this non-canonical pathway have yet to be described for excitable systems. Here we hypothesize anionic signaling lipids are a transducer of mechanical force to ion channels. Many ion channels and transporters are regulated by lipids and could serve as effector molecules of lipid raft disruption. Additionally, in eukaryotic membranes ${ }^{10,11}$ excess lipids and cellular cytoskeletal structures can resist tension on and propagation through plasma membranes ${ }^{12-15}$, suggesting the non-canonical disruption mechanism likely plays a prominent role for at least some ion channel activation. Linking atypical raft mechanosensation directly to a canonical 
mechanosensitive channel would constitute a distinct mechanism separate from the traditional "force from lipid" model typical of sensory systems where the channel directly senses stretch ${ }^{16,17}$.

TWIK-related $\mathrm{K}^{+}$channel type 1 (TREK-1) is a mechanosensitive member of the two-poredomain potassium (K2P) family that protects against pain ${ }^{18}$, and it is one of many channels regulated by lipids suggesting it could be down stream of raft disruption. Here we show membrane disruption releases PLD2 and generates the anionic lipid-signal phosphatidic acid (PA) that directly activates TREK-1. Blocking the synthesis of PA completely blocks stretch activation of TREK-1 in whole-cell patch-clamp electrophysiology. We conclude that in biological membranes, raft disruption and lipid mixing is a dominant mechanosensor for TREK-1 channels and the anionic lipid PA is an essential intermediary.

\section{RESULTS}

Mechanical disruption of lipid rafts in muscles cells.

The best studied raft domains contain saturated lipids, cholesterol, sphingomyelin, and monosialotetrahexosylganglioside1 (GM1) ${ }^{7}$. Cells contain a second class of lateral heterogeneity comprised of anionic lipids $\mathrm{PIP}_{2}$ and $\mathrm{PIP}_{3}$, which cluster $^{19-21}$ separate from GM1 domains and separate from each other ${ }^{5,22,23}$ (Supplemental Fig. S1a). Lipid domains are thought to be sub-100 nm diameter structures, may include protein interactions ${ }^{24}$, but even with super-resolution imaging techniques such as direct stochastic optical reconstruction microscopy (dSTORM) and stimulated emission depletion (STED) ${ }^{25-28}$ their exact structure remains controversial ${ }^{29,30}$. We previously used dSTORM to image livespontaneous and chemical disruption of GM1 domains in muscle cells ${ }^{5}$ leading us to asked if force induced disruption could directly be measured with imaging. Imaging direct disruption of lipid domains by force is important since GM1 and $\mathrm{PIP}_{2}$ lipids are part of all cellular membranes and force would be an endogenous disruptor of lipid order for most cell types.

To directly image force-induced disruption of lipid domains, we developed a process to chemically fix disrupted domains in the membrane of differentiated $\mathrm{C} 2 \mathrm{C} 12$ muscle cells (mouse myocytes) using a pump and shear chambers (ibidi $\mu$-Slide $\mathrm{I}^{0.4}$ parallel-plate) (Supplemental Fig. 2a). We fixed the cells with a combination of both paraformaldehyde and glutaraldehyde and confirmed by fluorescence recovery after photobleaching (FRAP) that are conditions inhibit extra-domain diffusion of GM1 lipids (Supplemental Fig. 2b). We tested muscle cells since they are known to respond to force and our previous experiments suggested force disrupts their GM1 domains ${ }^{5}$. Furthermore muscle cells endogenously express PLD and TREK-1 channels ${ }^{5,31}$ allowing for observation of their dynamics without overexpression artifacts.

First, we initiated domain disruption by pumping phosphate buffered saline (PBS) across the $\mathrm{C} 2 \mathrm{C} 12$ cells with a calibrated shear force of 3 dynes $/ \mathrm{cm}^{2}$, a physiologically relevant force to muscle cells ${ }^{5,31}$. Immediately after applying shear, we infused fixative agents to the shear buffer which allowed the cells to be fixed in a mechanicallystimulated state. Non-sheared control cells were grown and treated similarly on static coverslips without shear. Fixed cells, with and without shear, were then prepared for dSTORM imaging using antibodies or cholera toxin $\mathrm{B}(\mathrm{CtxB})$ with high affinity corresponding to either $\mathrm{PIP}_{2}$ or GM1 domains respectively ${ }^{5}$. The amount of fluorescent labeling remained relatively constant between shear and static cells (Supplemental Fig. 2c).

We found shear forces robustly disrupted both GM1 and $\mathrm{PIP}_{2}$ domain size in fixed $\mathrm{C} 2 \mathrm{C} 12$ cells (Fig. 1a-b). Interestingly, we observed that the shear specific disruption reduced GM1 domain size more than $\mathrm{PIP}_{2}$ domains. Prior to shear, the estimated size of GM1 domains were larger than $\mathrm{PIP}_{2}$ domains $(167 \pm 3$ vs. $154 \pm 1 \mathrm{~nm}$ in diameter). After shear, GM1 domains were smaller than $\mathrm{PIP}_{2}$ domains (131 \pm 3 vs. $139 \pm 1 \mathrm{~nm}$ in diameter, Fig. 1cd). Domain size was determined using imagebased clustering with identical threshold 

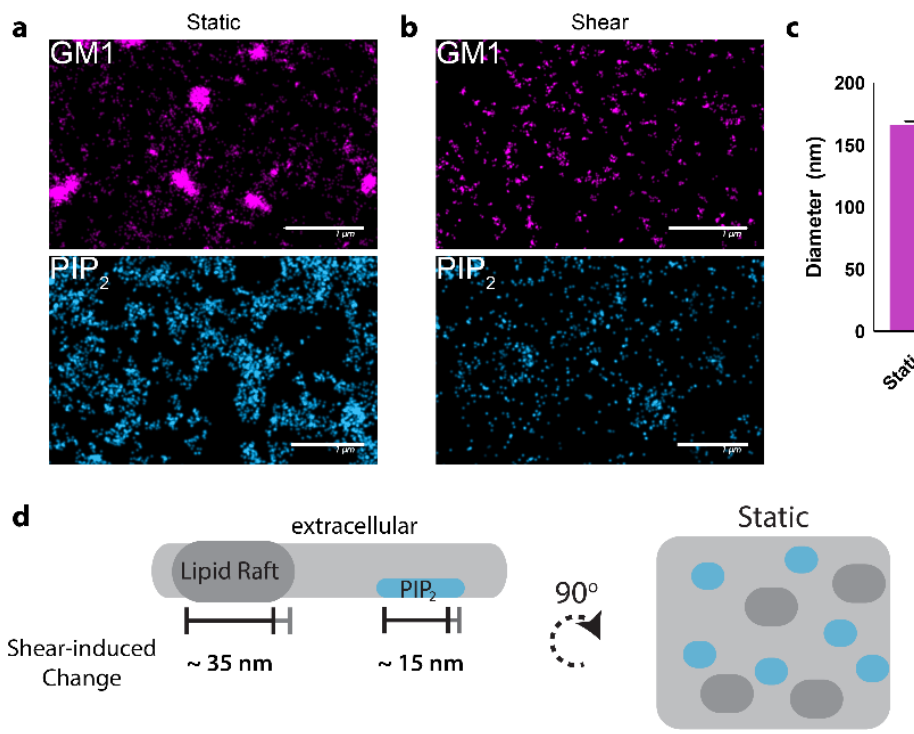

Figure 1. Mechanical induced disruption of $\mathrm{GM}_{1}$ and $\mathrm{PIP}_{2}$ domains.

(a-b) Representative images showing the effect of shear on the size of GM1 and PIP 2 labeled domains in muscle $\mathrm{C} 2 \mathrm{C} 12$ cells. When shear force is applied the apparent size of both domains decreases significantly (c) $\left(^{* *}\right.$ is $p<0.01,{ }^{* * * *}$ is $p<0.0001, n>800$ rafts from 5-6 cells). The dominant domain by area shifts from CTxB-labeled domains to $\mathrm{PIP}_{2}$ domains after shear is applied (d).

parameters for all images (see methods). The absolute size has potential artifacts from the analysis $^{32}$, however, the absolute size of rafts is inconsequential to our hypothesis. Rather, the change in raft size under otherwise identical conditions is important as it suggests force could directly affect palmitate-dependent protein localization to the GM1 domain and cause a translocation of PLD2 similar to chemical disruption ${ }^{5}$.

The cellular sensor of force in TREK-1 mechano-transduction.

PLD2 is palmitoylated at cysteines near its $\mathrm{PH}$ domain, which is required to localize it to lipid rafts ${ }^{33}$. The $\mathrm{PH}$ domain also binds $\mathrm{PIP}_{2}$ which opposes the localization by palmitoylation (Supplemental Fig. 1c). We previously demonstrated that chemical disruption of GM1 domains activates PLD2 by substrate presentation ${ }^{5}$ a result that is consistent with PLD regulation by caveolin ${ }^{34}$ and force induced flattening of caveolin ${ }^{4}$. After disruption, PLD2 rapidly translocates from GM1 to $\mathrm{PIP}_{2}$ domains where the enzyme mixes with its substrate phosphatidylcholine $(P C)$ to

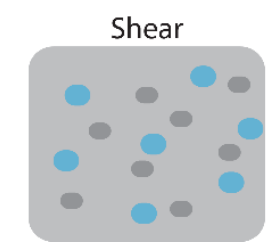

produce $\mathrm{PA}$. In addition to binding $\mathrm{PIP}_{2}$, PLD2 also binds directly to the Cterminus of TREK-1 and activates the channel through local production of phosphatidic acid $^{35}$ presumably in the disordered region of the cell membrane ${ }^{5}$. Previous work also implicated TREK-1 Cterminus in mechanosensitivity ${ }^{36}$.

We reasoned mechanical PLD2 activation could transduce a component of force to TREK-1 in a biological membrane independent of direct TREK-1 stretch. If PLD2 and PA are intermediaries in TREK-1 activation, this mechanism would link atypical raft mechanosensation directly to a canonical
mechanosensitive channel and support our proposed mixing and lipid-signaling mechanism for stretch activation. We estimated latency of PLD translocation to be very rapid $^{5}$ ( 650 $\left.\mu \mathrm{sec}\right)$ suggesting our proposed mixing mechanism is suitable for fast mechanical responses.

TREK-1 was previously shown to respond to mechanical force in cultured mammalian cells ${ }^{37}$. However, while TREK-1 is not endogenous to these cell types, PLD2 and GM1 domains are endogenous, and they are mechanosensitive ${ }^{5}$. However, prior to concluding TREK-1 must directly sense some type of curvature, tension, or forcefrom-lipid, a control experiment is needed that blocks PLD2 and shows the channel is still directly sensitive to force absent PLD2 activity, suggesting a control is needed to distinguish PLD2 mechanosensitivity from direct TREK-1 mechanosensitivity.

To distinguish the contribution of raft disruption from direct force acting on TREK-1, we tested TREK-1 currents with and without a catalytically dead K758R PLD2 mutant (xPLD2) ${ }^{38}$. The over expressed xPLD2 does not remove 
wildtype PLD from the membrane, but overexpressed XPLD2 is sufficiently high (>10 fold) ${ }^{39}$ to outcompete wildtype binding to TREK-1 thus acting as a pseudo dominant negative. As such, the contribution of TREK-1 activation arising from raft disruption pathway should be XPLD2 sensitive, i.e., XPLD2 should block raft dependent TREK-1 activation. In contrast, the direct force from lipid (i.e. the membrane tension) acting on the channel should be PLD2 independent, resulting in TREK-1 stretch-activated current in the presence of XPLD2.

Consistent with previous experiments, overexpression of TREK-1 with wildtype or endogenous PLD2 led to a robust TREK-1 pressure-dependent current in HEK 293 cells (Fig. 2a). However, overexpression of xPLD2 inhibited all detectible stretch-activated TREK-1 current (Fig. 2b-c). This suggests that all detectable TREK-1 mechanosensitive current in HEK cells is PLD-dependent and an assumption that no endogenous proteins are contributing to the mechanotransduction pathway is likely incorrect. The PLD dependent current from TREK-1 is highly mechanosensitive responding to negative pressure (0 to $-60 \mathrm{mmHg}$ ) with a half maximal pressure $\left(P_{50}\right)$ of $\sim 32$ $\mathrm{mmHg}$ and yielding up to $200 \mathrm{pA}$ of TREK-1 current (Fig. 2a and Supplemental Fig. S3a-b) consistent with previous studies $^{40,41}$. The inhibition of current was highly significant with or without overexpression of PLD2 $\quad(p<0.002$ and 0.007 respectively, Fig. 2c). Furthermore, the $\mathrm{P}_{50}$ significantly decreased from 37 to $31 \mathrm{mmHg}$ a

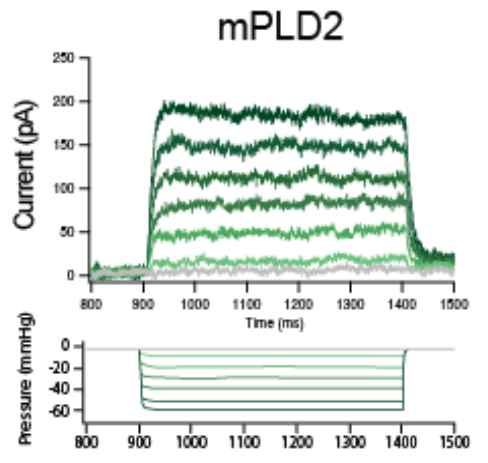

C

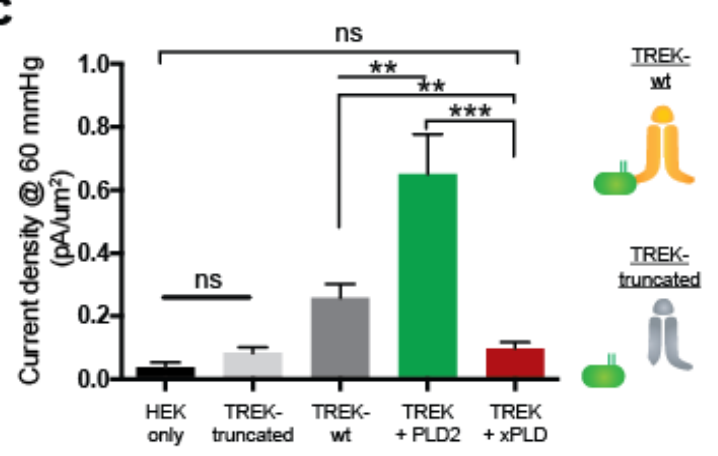

f

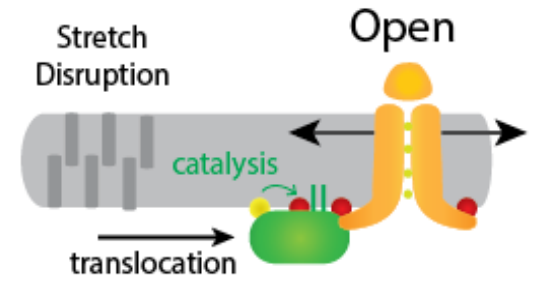

b
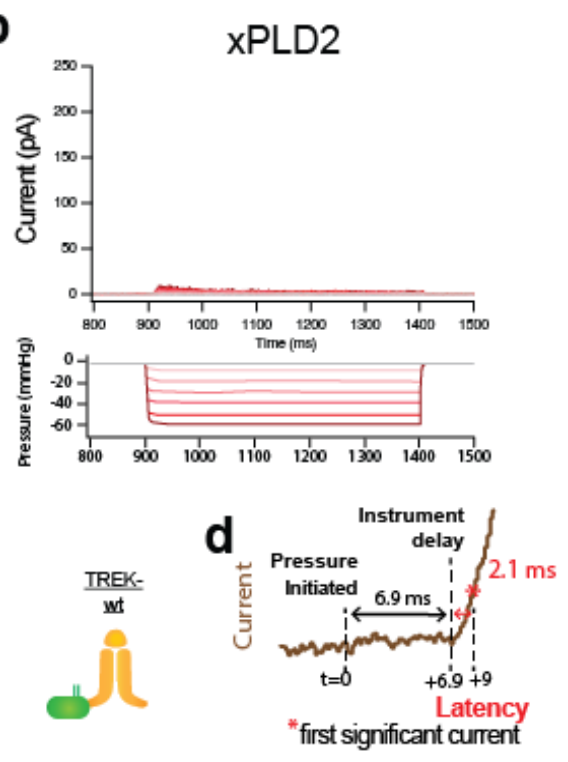

e

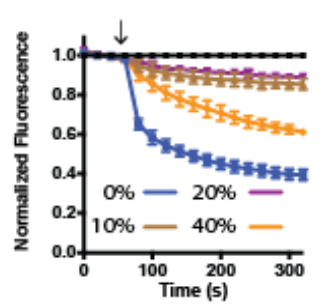

Closed
Figure 2. Domain disruption mediates stretch-activation of TREK-1 channels. (a-b) Representative traces of TREK currents with PLD (green, left) and XPLD (red, right) overexpression showing the near elimination of current when PLD is not active. (c) Channel response to pressure clamp with TREK-1 was greater than that with overexpression with xPLD $(p<0.007, n=16-23)$, and was enhanced by the overexpression of PLD2 $(p<0.002, n=15-23)$. There was no significant difference between wt HEK cells, TREK with XPLD, and a truncated version of TREK which lacks the PLD binding site (see side panels) indicating that PLD is required for TREK mechanical stimulation ( $p>0.05, n=5-16)$. (d) PLD2 dependent TREK-1 currents measured by patch clamp electrophysiology showing the latency of lipid mixing. Instrument latency is the time the clamp requires to reach $60 \mathrm{mmHg}$ and propagation the pressure to the pipette $(6.9 \mathrm{~ms})$. The channel current trends towards opening almost immediately after the instrument delay and the current becomes statistically significant (shown by asterisk) by $2.1 \mathrm{~ms}$ although current it observed almost immediately after pressure reaches the patch indicating he activation likely occurs much faster than the reported time. (e) Flux assay of reconstituted TREK-1 into vesicles show that TREK-1 is sensitive to the thickness of the membrane as shown by adding varying amounts of cholesterol to azolectin membranes. (f) During stretch disruption force is applied directly to the channel via FFL and domains are disrupted. Without catalysis of lipid by PLD, the contribution of FFL is shown to be insianificant to TREK-1 activitv in vivo. 
when PLD2 was overexpressed (Supplemental Fig. 3b). Our stretch activated currents were measured using a pressure clamp in the inside-out patch configuration similar to previous experiments (see methods) allowing for direct comparison to these previous studies.

To further confirm that PLD2 is responsible for stretch activation of TREK-1 currents in cellular membranes, we truncated the C-terminus of TREK-1 (truncated TREK-1) and compared its pressure activation to wildtype-PLD2 binds to the C-terminus of TREK ${ }^{35}$ (Supplemental Fig. S3c). As expected, C-terminally truncated TREK-1 was almost completely insensitive to stretch activation by pressure with no significant difference between it and current measured in non-transfected cells (Fig. 2c and raw traces, Supplemental Fig. S3a).

For lipid mixing to act as a relevant mechanosensor for an ion channel, latency (response time) needs to be fast, (i.e. $\mu$ s to low ms time frame). To determine the latency for PLD2 based lipid mixing we measured the delay time for PLD2 specific stretch activation of TREK-1 with pressure clamp. This is possible since all the observed stretch activated TREK-1 current was PLD2 dependent (Fig. 2c). Our instrument setup has a $6 \mathrm{~ms}$ delay (4 $\mathrm{ms}$ in the instrument and 2.9 $\mathrm{ms}$ in the connectors; see methods). After instrument delay we see initial TREK-1 currents almost immediately and the current reaches statistical significance within $2.1 \mathrm{~ms}$ at $60 \mathrm{mmHg}$ (Fig. 2d), demonstrating PLD2 lipid mixing is capable of latencies suitable for fast activation of a mechanosensitive channel.

We also found that purified TREK-1 in liposomes show major regulation by the thickness of the membrane in which TREK-1 resides (Fig. 2e) as well as by anionic lipid ${ }^{42}$. These results show in a purified system how PLD2 is the primary force transducer for TREK-1 activation in HEK cell membranes (see Fig. 2f). Since domain disruption can lead to a change in thickness and PLD activation by raft disruption is known to occur ${ }^{5}$, we looked to observe if TREK-1 responded to shear by translocating similar to PLD2.

The TREK-1 force transduction pathway.
We previously showed PLD2 responds directly to shear force using a live PLD2 shear assay. 3 dynes $/ \mathrm{cm}^{2}$ shear force robustly increased PLD activity measured in real time ${ }^{5}$. Here we investigate protein translocation in response to mechanical force. We directly imaged lipid rafts, TREK-1, and PLD2 localization using dSTORM in C2C12 cells. PLD2 and TREK are endogenously expressed in $\mathrm{C} 2 \mathrm{C} 12$ cells. We applied a force of 3 dynes $/ \mathrm{cm}^{2}$ mechanical shear to cells and determined lipid mixing by monitoring fluorescently labeled PLD2 and TREK-1 localization with GM1 and $\mathrm{PIP}_{2}$ and each other. Pair correlation was used to determine localization. Pair correlation is not affected by oversampling, thus making it appropriate for stochastic methods such as dSTORM $^{32,43}$.

Prior to shear, PLD2 was robustly localized with GM1 domains and moderately with $\mathrm{PIP}_{2}$ domains as shown previously ${ }^{5}$ while TREK-1 was most strongly associated with $\mathrm{PIP}_{2}$ domains (Fig. 3a-c). TREK-1 also localized with PLD2 (Fig. 3d) as expected since PLD is known to bind directly to TREK $-1^{35}$. TREK-1 localized minimally with GM1 domains, a result consistent with a proportion of TREK-1 preferring the disordered, bulk-like lipids when not bound to $\mathrm{PLD}^{42}$. Images of each individual channel for the abovementioned localizations are included in Supplemental Fig. 4.

After mechanical shear, PLD2 robustly translocated from GM1 to $\mathrm{PIP}_{2}$ domains (Fig. 3ab). Shear-induced ejection of PLD2 from GM1 domains was almost complete-more robust than chemical disruption ${ }^{5}$. PLD2 and TREK-1 colocalization remained roughly constant after shear (Fig. 3d) suggesting the two proteins shift as a complex towards $\mathrm{PIP}_{2}$ domains (unsaturated lipids). We co-labeled TREK-1 and PLD2consistent with this model, TREK-1 also shifted toward $\mathrm{PIP}_{2}$ domains after shear (Fig. 3c). Localization between TREK-1 and GM1 domains does not change (Supplemental Fig 5) unless cholesterol is present. In a companion study we show cholesterol increases TREK-1 localization to GM1 domains in primary neurons and shear disrupts that interaction (Neyabosadri et al, Figure $4 A-B)$. Together this suggests a model whereby the PLD2-TREK-1 complex translocates from GM1 domains towards $\mathrm{PIP}_{2}$ domains where PLD2 is 
active, allowing for activation of TREK-1 ${ }^{35}$ (Fig. 2, 3e). Since $\mathrm{PIP}_{2}$ directly antagonizes TREK-1 ${ }^{44,45}$, the localization of TREK-1 to $\mathrm{PIP}_{2}$ before and after shear (Fig 3c,e) explains the strong inhibition of TREK-1 by XPLD2 (Fig. 2b-c).

\section{Mechanical disruption} of lipid rafts in neurons.

To establish our mechanical mechanism of domain disruption in a neuronal system, we tested mechanical shear in neuroblastoma 2a (N2A) cells, a generic mammalian neuronal line. Among cells, nerves can be particularly sensitive to mechanical force and play an important role generating pain and touch sensation in animals. PLD and lipid partitioning are found in all types of cells including neurons ${ }^{46}$.

$\mathrm{N} 2 \mathrm{a}$ cells were found to recapitulate both the domain disruption and PLD2 activation in response to mechanical shear as was observed in muscle cells. Application of 3 dynes $/ \mathrm{cm}^{2}$ lead to a significant decrease in the relative size of the labeled domains in $\mathrm{N} 2 \mathrm{a}$ cells of $\sim 50 \mathrm{~nm}$ in diameter (Fig. 4a-b). This decrease also lead to an increase in the activity of PLD2 (Supplemental Fig. 6a) as would be predicted by our previous findings in muscle cells. These findings show that the mechanism is not
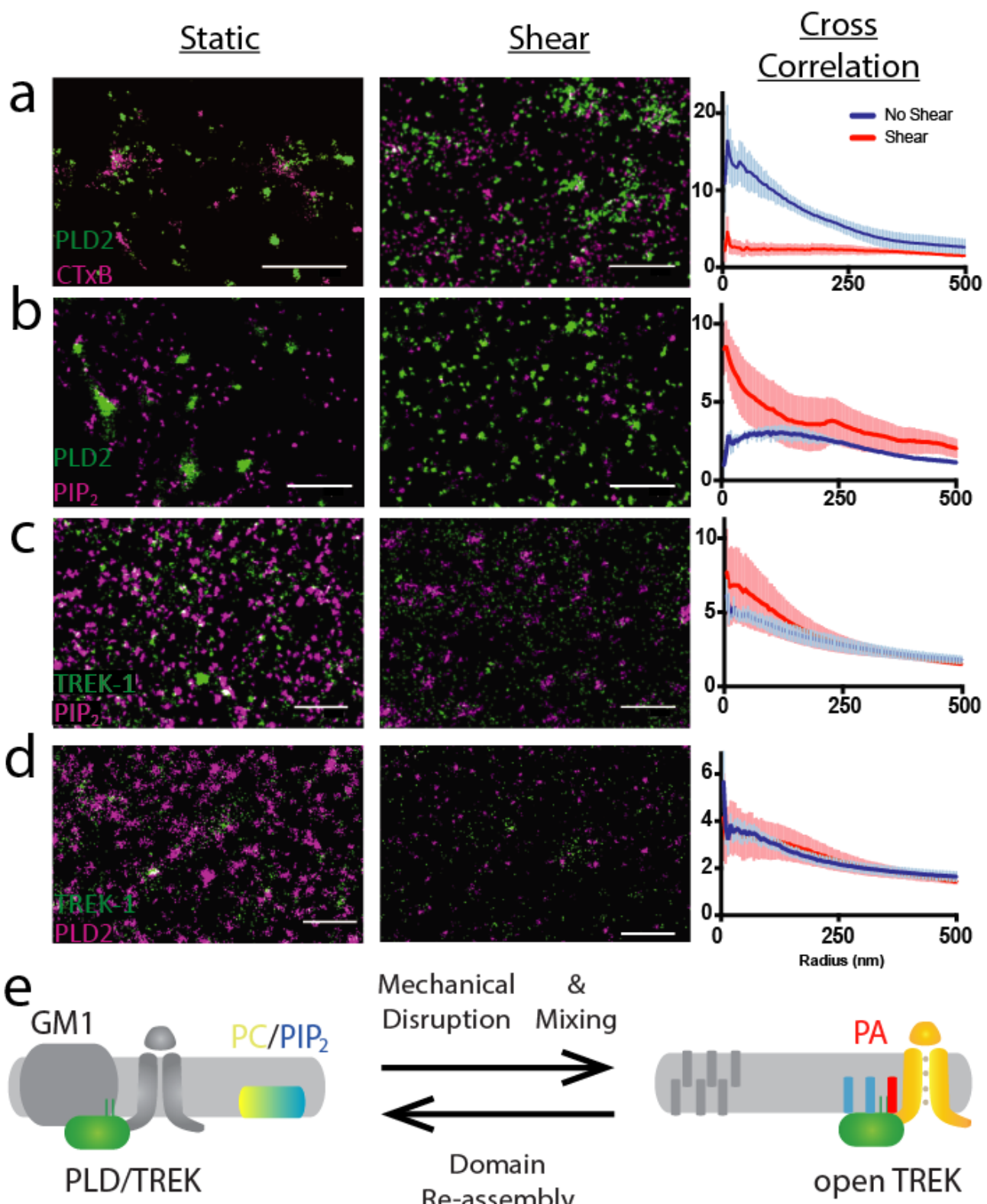

Figure 3. Shear induces protein translocation and lipid mixing.

(a-c) dSTORM images without shear (left) and with 3 dynes $/ \mathrm{cm} 2$ shear forces (middle) along with cross correlation showing the localization changes due to shear (right). Due to their high clustering before shear, CTXB and PLD2 show a sharp decrease in crosscorrelation after shear (a). Mechanical disruption increases the localization between $\mathrm{PI}(4,5) \mathrm{P}_{2}$ and both PLD2 (b) and TREK-1 (c). (d) PLD and TREK show no change in localization, likely indicating a complex between hem remains intact after shear. (e) illustrates how after mechanical stimulus, lipid microdomains (dark grey) are disrupted, allowing the PLD2-TREK-1 complex to translocate to $\mathrm{PI}(4,5) \mathrm{P}_{2}$ domains (blue).

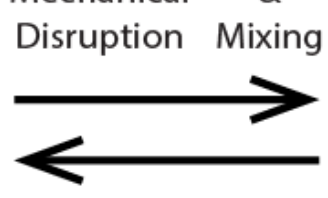

Domain

Re-assembly
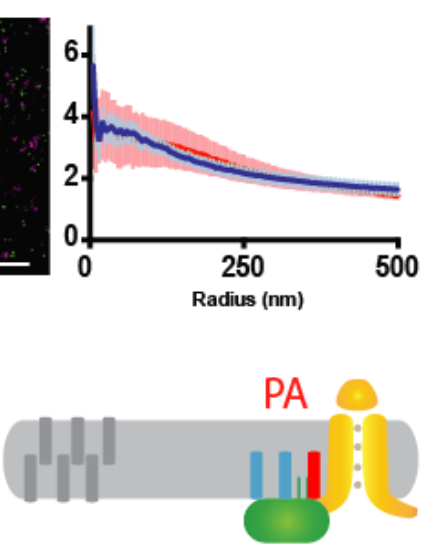

open TREK

constrained to muscle cells and likely is activated in any cells which contain both lipid domains and express PLD2. 
Regulation of mechano/pain thresholds in vivo

We then reasoned that if domain regulation of lipids were a common mechanism of mechanosensation than evidence of this mechanism could be observed in diverse animal species. We thus chose D. melanogaster (fruit flies) due to both its possession of a PLD2 ortholog as well as its evolutionary distance from the mammalian cell lines used previously. We found that GM1 domains exist in the brain of flies (Fig. 4c) and that they can be disrupted ${ }^{39}$. Observation of the activity of the PLD ortholog in neuronallyderived cell lines (BG2-c2) also showed sensitivity to mechanical shear forces (Fig. 4d), consistent with regulation through raft disruption. We wanted to observe the behavior of these animals in order to determine if there was a physiological contribution from PLD to the mechanosensitivity of the whole animal. One behavior which could show this contribution is an arousal-assay which measures the amount of mechanical stimulation needed to excite a fly into movement from rest. If PLD-synthesized PA is also upstream of mechanosensitive potassium channels in flies (as in mammalian cells) we would predict a decrease in the amount of force needed to cause fly arousal.

Using single-animal measurements of arousal threshold ${ }^{47,48}$ (see Supplemental Fig. 6b), we examined whether PLD could regulate fly mechanosensation. Flies without functional PLD (PLD $\left.{ }^{\text {null }}\right)$ were subjected to a series of incremental vibrational stimuli every 30 minutes for 24 hours. For each series, the level of stimulation required to arouse the fly, indicated by motion, was recorded using automated machine vision tracking. Averages over the 24 hours were compared to genetically matched controls. PLD ${ }^{\text {null }}$ flies showed a significantly lower arousal threshold than their control strains, indicating increased sensitivity to mechanical force (Fig. 4e). We further used a neuronal-specific driver, Nsyb GAL4, combined with a PLD RNAi line (PLD-KD) and obtained a similar result, indicating that the phenotype is neuronal-specific (Fig. 4f). PLD also regulated responses to noxious pain stimuli (Supplemental Fig. 6c-e).

\section{DISCUSSION}

Our data demonstrate, in a biological membrane, lipid domain disruption is sensor that communicates force to the canonical mechanosensitive channel TREK-1 (Fig. 4g). The necessity of functional PLD2 for TREK-1 mechanosensitivity (Fig. 2) in HEK cells suggests the enzyme can dominate as the primary transducer and TREK-1 as a secondary transducer in the membranes of cultured cells. Hence the membrane first elicits an enzyme-mediated chemical signal and then the chemical signal is converted into an electrical signal by the channel. In theory, PLD2 could be activated by mechanical force and raise the global PA levels sufficient to activate TREK-1, however, truncated TREK-1 lacking the PLD localization domain (Fig. 2c) failed to respond and suggests that PLD2 localization is a significant contributor to TREK-1 activation. Since the PLD-TREK-1 complex was shown above to translocate to $\mathrm{PIP}_{2}$ domains and $\mathrm{PIP}_{2}$ is a TREK1 antagonist ${ }^{44}$, it is likely that without co-localization the local concentration of PA does not reach sufficient levels to compete with PIP2 binding ${ }^{11}$. It may be possible if PLD2 dependent PA production could reach activating concentration if the membrane and or cytoskeleton were sufficiently disrupted.

Conservative estimates of the latency of TREK-1 activation by chemical transduction through PLD2 is less than $2.1 \mathrm{~ms}$. In reality, the latency could be much faster, as the 2.1 ms latency is likely an upper limit due to the error in our instrument delay, not PLD2 mixing-TREK-1 current appeared to rise immediately after the instrument delay (Fig. 2e). Regardless, the $2.1 \mathrm{~ms}$ upper limit is safely within previously established mechanosensitive current latency ${ }^{49}$, suggesting that domain disruption is suitable mechanism for most mechano-transduction pathways. This direct measurement in HEK293 cells roughly agree with the $650 \mu$ s theoretical latency previously estimated based on spatial separation of GM1 and $\mathrm{PIP}_{2}$ domains in $\mathrm{C} 2 \mathrm{C} 12$ cells $^{5}$.

In artificial membranes TREK-1 responds directly to membrane thinning ${ }^{42}$ and stretch $^{41,50}$, i.e. FFL appears to directly open and close the channel $^{17,51}$. In contrast, in a biological membrane, our electrophysiology experiments, imaging, and 
enzyme assay clearly show that anionic lipid production by PLD2 is necessary for mechanical TREK activity in HEK cells (Fig. $4 \mathrm{~g}$ )-no amount of stretch was able to substantially activate the channel absent PLD2 (Fig. 2b-d and Supplemental Fig. S3a).

$$
\text { In }
$$

physiological system the two variables, thickness and PA, likely act as coincidence detectors, allowing for careful control over the hyperpolarization effect of TREK-1. It is likely that the translocation of TREK-1 from GM1 domains to the bulk membrane facilitates the thickness change required for activation of the channel since the thickness differential is $\sim 30 \% \quad(12 \AA)$, while pressure-mediated

thinning can only change the thickness $<4 \% \quad(1.5 \AA)$ before resulting in lysis ${ }^{15}$. The hydrophobic mismatch between the protein and lipid after translocation likely provides the curvature speculated to activate the channel by FFL. PA production could also directly alter the curvature, thickness, or additional signaling enzymes near TREK-1, but these putative pathways would not significantly change the finding that PLD2 is a
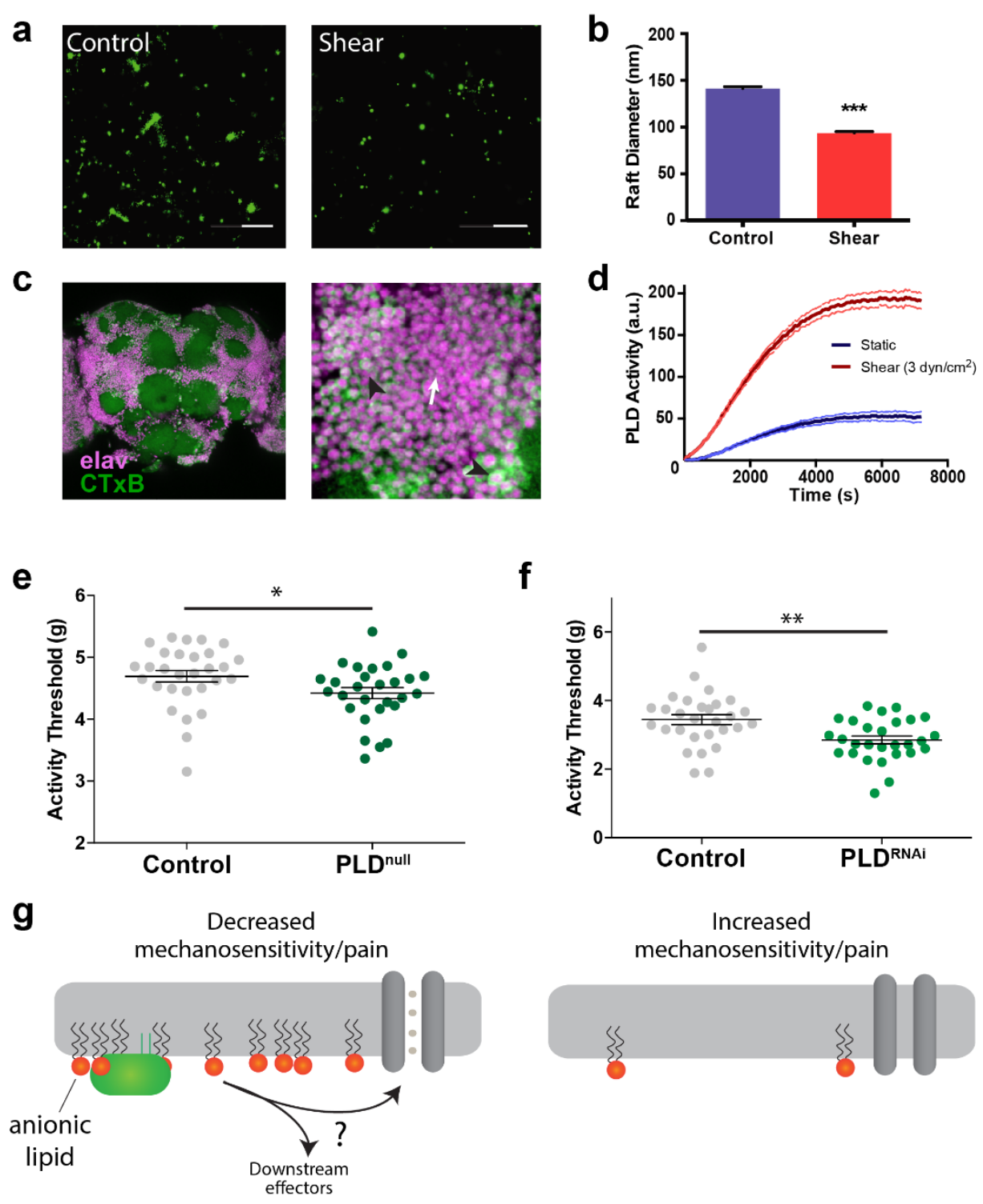

Increased mechanosensitivity/pain

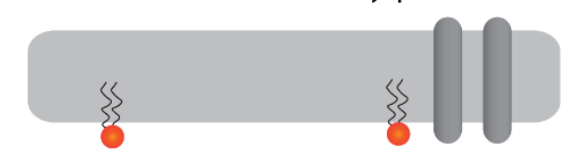

Figure 4. PLD is regulated in neurons and shifts the mechanosensitivity of $D$. melanogaster.

(a-b) Lipid domains regulate the activity of PLD in neuronal cells. CTxB labeled domains (a) decrease in size from 141 to $92 \mathrm{~nm}$ after application of 3 dynes $/ \mathrm{cm}^{2}$ of shear force (b). Scale bar is $1 \mu \mathrm{m}$. (c) GM1 lipids are found in D. melanogaster. The CTxB labeling shows that GM1 is found throughout the brain (left). Zoomed section (right) shows that most of the labeling is found on the membrane. There are notable variations in the amount of CTxB labeling with some cells expressing GM1 over the entire membrane (black arrows) while some cells only have labeling in small puncta (white arrow). (d) The activation of PLD observed previously in muscle cells is recapitulated in drosophila neurons indicating that PLD is regulated similarly in both cell types. (e-f) Interrogation of the role of PA in mechanosensation in $D$. melanogaster. (e) show that removal of PLD function results in a decreased threshold of activation than the genetically matched controls $(w 1118)(p=0.039, n=28-29)$, consistent with the prediction that $P A$ is upstream of hyperpolarizing potassium channels. (f)The same result was observed in a PLD ${ }^{\mathrm{RNAi}}$ line which results in PLD knockdown only in the neurons of Drosophila $(\mathrm{p}=0.002, \mathrm{n}=28-29)$. (g) Proposed general mechanism. Upon microdomain disruption, PLD is activated (left) and catalyzes various anionic lipid products. These products act on channels and other effectors to control thresholds of stimulation. Without PLD2 (right) mechanically derived anionic lipid is not made, resulting in the thresholds of mechanosensitivity and pain to be decreased. 
primary transducer for TREK-1 activation in a cellular membrane. The hydrophobic mismatch component of TREK-1 activation is also seen in prokaryotic mechanosensors ${ }^{52}$. The effect of translocation and lipid presentation is not known for prokaryotes.

Raft disruption may directly affect many ion channels since palmitoylation alone is sufficient to target proteins to GM1 domains ${ }^{6}$ and many ion channels are palmitoylated ${ }^{53}$ and respond to lipid binding or changes in the lipid environment. In theory, mechanical disruption of GM1 domains could cause translocation of any palmitoylated channel in a single step, directly exposing the channel to an activating lipid. For example, the voltage-activated sodium channel $\left(\mathrm{Na}_{\mathrm{v}}\right) 1.9$ is localized to lipid domains and domain disruption (by chemically removing cholesterol) induces channel translocation out of GM1 domains and a corresponding pain response ${ }^{54}$. In such cases, entering membrane domains containing disordered lipids may be sufficient to activate the channel.

Disruption of palmitate-mediated localization may be a common mechanism for activating transduction pathways; many important signaling molecules are palmitoylated including tyrosine kinases, GTPases, CD4/8, and almost all G-protein alpha subunits ${ }^{55}$. Translocation of these proteins, or a palmitoylated binding partner, from lipid domains by mechanical force could alter their available substrates and affect downstream signaling. Since many mechanosensitive channels are either regulated or directly gated by lipids, it is likely that other channels are downstream effectors of domain disruption in biological membranes. For example, some transient receptor potential (TRP) channels may be activated by domain disruption since they are regulated by lipid rafts ${ }^{56}$ and function downstream of anionic lipid signaling ${ }^{57}$.

While fly orthologs of TREK-1 exist, it is not clear which of these may bind PLD, greatly hampering research into the downstream pathway of PLD in the fly. More work will need to be done to determine the native binding partner of PLD, and thus PA, in D. melanogaster. This said, since leak potassium channels, such as TREK-1, are typically protective against pain i.e. they hyperpolarize the cell $^{58}$, we expect PA synthesis should decrease sensitivity to pain in flies, which is consistent with the result we observed in the PLD deficient flies.

Lastly, PA's regulation of $D$. melanogaster mechanosensation and pain (Fig. 4) adds in vivo support to a growing list of anionic lipids that set force sensing thresholds. For example, $\mathrm{PIP}_{2}$ sets the threshold for mechanical B-cell activation ${ }^{59}$. Likewise, sphingosine-1-phosphate (S1P), an anionic lipid similar to PA, regulates pain in mice ${ }^{60}$. PLD's activation by mechanical force and substrate presentation helps explain how anionic lipids could directly set pain thresholds and mechanosensitivity via canonical mechanosensitive ion channels. A single lipid regulating multiple channels simultaneously is much more likely to affect a whole animal than a single channel.

\section{ACKNOWLEDGEMENTS}

We thank Tamara Boto and Seth Tomchik for their assistance in the Drosophila shock experiments, Michael Frohman from Stony Brook for the mouse PLD and mutant PLD cDNA, Steven Long from Memorial Sloan Kettering for human TREK-1-GFP, Padinjat Raghu for the PLD mutant Drosophila, Andrew S. Hansen for PLD experiments, multiple aspects of experimental design and discussion, Yul Young Park for the electrophysiology experimentation, and Carl Ebeling for his help and discussion on the imaging analysis. This work was supported by a Director's New Innovator Award to S.B.H. (1DP2NS087943-01) from the National Institutes of Health, an R01 to W.W.J. (R01AG045036) from the National Institute on Aging, and a graduate fellowship from the Joseph B. Scheller \& Rita P. Scheller Charitable Foundation to E.N.P. The authors declare no conflict of interest.

\section{AUTHOR CONTRIBUTIONS}

Conceptualization, E.N.P. and S.B.H.; Methodology, E.N.P., M.G., K.R.M, and S.B.H; Investigation, E.N.P., M.G., and K.R.M; Resources, W.W.J., E.M.J., and S.B.H.; Writing Original Draft, E.N.P. and S.B.H.; Writing - Review and Editing, E.N.P., M.G., M.A.P, E.M.J., and 
S.B.H.; Supervision, W.W.J. and S.B.H.; Funding Acquisition, W.W.J. and S.B.H.

\section{WORKS CITED}

1. Ranade, S. S., Syeda, R. \& Patapoutian, A. Mechanically Activated Ion Channels. Neuron 87, 1162-79 (2015).

2. Julius, D. Molecular mechanisms of nociception . Nature 413, 203-210 (2001).

3. Hahn, C. \& Schwartz, M. A. Mechanotransduction in vascular physiology and atherogenesis. Nat Rev Mol Cell Biol 10, 53-62 (2009).

4. Sinha, B. et al. Cells respond to mechanical stress by rapid disassembly of caveolae. Cell 144, 402-413 (2011).

5. Petersen, E. N., Chung, H.-W., Nayebosadri, A. \& Hansen, S. B. Kinetic disruption of lipid rafts is a mechanosensor for phospholipase D. Nat. Commun. 7, 13873 (2016).

6. Levental, I., Lingwood, D., Grzybek, M., Coskun, U. \& Simons, K. Palmitoylation regulates raft affinity for the majority of integral raft proteins. Proc. Natl. Acad. Sci. 107, 22050-22054 (2010).

7. Lingwood, D. \& Simons, K. Lipid rafts as a membrane-organizing principle. Science 327, 46-50 (2010).

8. Loh, J. et al. Acute decrease in plasma membrane tension induces macropinocytosis via PLD2 activation. J. Cell Sci. (2019). doi:10.1242/jcs.232579

9. Lin, S.-S. \& Liu, Y.-W. Mechanical Stretch Induces mTOR Recruitment and Activation at the Phosphatidic Acid-Enriched Macropinosome in Muscle Cell. Front. Cell Dev. Biol. 7, 78 (2019).

10. Hansen, S. B. Lipid agonism: The PIP2 paradigm of ligand-gated ion channels. Biochim. Biophys. Acta 1851, 620-628 (2015).

11. Robinson, C. V., Rohacs, T. \& Hansen, S. B. Tools for Understanding Nanoscale Lipid Regulation of Ion Channels. Trends Biochem. Sci. (2019). doi:10.1016/j.tibs.2019.04.001

12. Raucher, D. \& Sheetz, M. P. Characteristics of a Membrane Reservoir Buffering Membrane Tension. Biophys. J. 77, 1992-2002 (1999).

13. Gauthier, N. C., Masters, T. A. \& Sheetz, M. P.
Mechanical feedback between membrane tension and dynamics. Trends Cell Biol. 22, 527-35 (2012).

14. Shi, Z., Graber, Z. T., Baumgart, T., Stone, H. A. \& Cohen, A. E. Cell Membranes Resist Flow. Cell 175, 1-11 (2018).

15. Hamill, O. P. \& Martinac, B. Molecular Basis of Mechanotransduction in Living Cells. Physiol. Rev. 81, 685-740 (2001).

16. Haswell, E. S., Phillips, R. \& Rees, D. C. Mechanosensitive channels: what can they do and how do they do it? Structure 19, 1356-69 (2011).

17. Teng, J., Loukin, S., Anishkin, A. \& Kung, C. The force-from-lipid (FFL) principle of mechanosensitivity, at large and in elements. Pflügers Arch. Eur. J. Physiol. 467, 27-37 (2015).

18. Honoré, E. The neuronal background K2P channels: focus on TREK1. Nat. Rev. Neurosci. 8, 251-261 (2007).

19. Graber, Z. T., Shi, Z. \& Baumgart, T. Cations induce shape remodeling of negatively charged phospholipid membranes. Phys. Chem. Chem. Phys. 19, 15285-15295 (2017).

20. Sarmento, M. J., Coutinho, A., Fedorov, A., Prieto, M. \& Fernandes, F. Ca(2+) induces PI(4,5)P2 clusters on lipid bilayers at physiological PI(4,5)P2 and $\mathrm{Ca}(2+)$ concentrations. Biochim. Biophys. Acta 1838, 822-30 (2014).

21. Wang, Yu-hsiu; Collins, Agnieszka; Guo, Lin; SmithDupont, Kathryn B.; Gai, Feng; Svitkina, Tatyana; Janmey, P. A. Divalent cation-induced cluster formation by polyphosphoinosited in model membranes. J Am Chem Soc. 134, 3387-3395 (2012).

22. van den Bogaart, G. et al. Membrane protein sequestering by ionic protein-lipid interactions. Nature 479, 552-5 (2011).

23. Wang, J. \& Richards, D. a. Segregation of PIP2 and PIP3 into distinct nanoscale regions within the plasma membrane. Biol. Open 1, 857-62 (2012).

24. Honigmann, A. et al. Scanning STED-FcS reveals spatiotemporal heterogeneity of lipid interaction in the plasma membrane of living cells. Nat. Commun. 5, (2014).

25. Jones, S. a, Shim, S.-H., He, J. \& Zhuang, X. Fast, three-dimensional super-resolution imaging of live 
cells. Nat. Methods 8, 499-508 (2011).

26. Huang, B., Wang, W., Bates, M. \& Zhuang, X. Threedimensional super-resolution imaging by stochastic optical reconstruction microscopy. Science 319, 810-3 (2008).

27. Betzig, E. et al. Imaging intracellular fluorescent proteins at nanometer resolution. Science $\mathbf{3 1 3}$, 1642-1645 (2006).

28. Hess, S. T., Girirajan, T. P. K. K. \& Mason, M. D. Ultra-high resolution imaging by fluorescence photoactivation localization microscopy. Biophys. J. 91, 4258-4272 (2006).

29. Moon, S. et al. Spectrally Resolved, Functional Super-Resolution Microscopy Reveals Nanoscale Compositional Heterogeneity in Live-Cell Membranes. J. Am. Chem. Soc. 139, 10944-10947 (2017).

30. Sezgin, E., Levental, I., Mayor, S. \& Eggeling, C. The mystery of membrane organization: composition, regulation and roles of lipid rafts. Nat. Rev. Mol. Cell Biol. 18, 361-374 (2017).

31. Schneck, D. J. Mechanics of Muscle. Dynamic Behaviour of Materials Vol 1 (New York University Press, 1992). doi:10.1007/978-3-319-00771-7-1

32. Veatch, S. L. et al. Correlation functions quantify super-resolution images and estimate apparent clustering due to over-counting. PLoS One 7, (2012).

33. McDermott, M., Wakelam, M. J. O. \& Morris, A. J. Phospholipase D. Biochem. Cell Biol. 82, 225-53 (2004).

34. Czarny, M., Lavie, Y., Fiucci, G. \& Liscovitch, M. Localization of phospholipase $D$ in detergentinsoluble, caveolin-rich membrane domains: Modulation by caveolin-1 expression and caveolin. J. Biol. Chem. 274, 2717-2724 (1999).

35. Comoglio, Y. et al. Phospholipase D2 specifically regulates TREK potassium channels via direct interaction and local production of phosphatidic acid. Proc. Natl. Acad. Sci. U. S. A. 111, 13547-52 (2014).

36. Chemin, J. et al. Regulation of the Mechano-Gated K 2P Channel TREK-1 by Membrane Phospholipids. (2007). doi:10.1016/S1063-5823(06)59007-6

37. Chemin, J. et al. A phospholipid sensor controls mechanogating of the K+ channel TREK-1. EMBO J.
24, 44-53 (2005).

38. Toschi, A. et al. Regulation of mTORC1 and mTORC2 complex assembly by phosphatidic acid: competition with rapamycin. Mol. Cell. Biol. 29, 1411-20 (2009).

39. Pavel, M. A., Petersen, E. N., Lerner, R. A. \& Hansen, $S$. B. Studies on the mechanism of general anesthesia. bioRxiv (2018). doi:doi.org/10.1101/313973313973

40. Patel, A. J. et al. A mammalian two pore domain mechano-gated S-like K+ channel. EMBO J. 17, 4283-90 (1998).

41. Brohawn, S. G., Su, Z. \& Mackinnon, R. Mechanosensitivity is mediated directly by the lipid membrane in TRAAK and TREK1 K+ channels. Proc. Natl. Acad. Sci. U. S. A. 111, 3614-9 (2014).

42. Nayebosadri, A., Petersen, E. N., Cabanos, C. \& Hansen, S. B. A Membrane Thickness Sensor in TREK-1 Channels Transduces Mechanical Force. SSRN Electron. J. (2018). doi:10.2139/ssrn.3155650

43. Sengupta, P. \& Lippincott-Schwartz, J. Quantitative analysis of photoactivated localization microscopy (PALM) datasets using pair-correlation analysis. BioEssays 34, 396-405 (2012).

44. Cabanos, C., Wang, M., Han, X. \& Hansen, S. B. A Soluble Fluorescent Binding Assay Reveals PIP2 Antagonism of TREK-1 Channels. Cell Rep. 20, 12871294 (2017).

45. Chemin, J. et al. Up- and down-regulation of the mechano-gated K2P channel TREK-1 by PIP2 and other membrane phospholipids. Pflugers Arch. Eur. J. Physiol. 455, 97-103 (2007).

46. Simons, K. \& Toomre, D. Lipid rafts and signal transduction. Nat. Rev. Mol. Cell Biol. 1, 31-9 (2000).

47. Murphy, K. R., Park, J. H., Huber, R. \& Ja, W. W. Simultaneous measurement of sleep and feeding in individual Drosophila. Nat. Protoc. 12, 2355-2366 (2017).

48. Murphy, K. R. et al. Postprandial sleep mechanics in Drosophila. Elife 5, 1-19 (2016).

49. Christensen, A. P. \& Corey, D. P. TRP channels in mechanosensation: direct or indirect activation? Nat. Rev. Neurosci. 8, 510-21 (2007). 
50. Berrier, C. et al. The purified mechanosensitive channel TREK-1 is directly sensitive to membrane tension. J. Biol. Chem. 288, 27307-14 (2013).

51. Anishkin, A., Loukin, S. H., Teng, J. \& Kung, C. Feeling the hidden mechanical forces in lipid bilayer is an original sense. Proc. Natl. Acad. Sci. 111, 78987905 (2014).

52. Kung, C. A possible unifying principle for mechanosensation. Nature 436, 647-54 (2005).

53. Shipston, M. J. Ion channel regulation by protein palmitoylation. J. Biol. Chem. 286, 8709-8716 (2011).

54. Amsalem, M., Poilbout, C., Ferracci, G., Delmas, P. \& Padilla, F. Membrane cholesterol depletion as a trigger of Nav1.9 channel-mediated inflammatory pain. EMBO J. 37, e97349 (2018).

55. Aicart-Ramos, C., Valero, R. A. \& Rodriguez-Crespo, I. Protein palmitoylation and subcellular trafficking. Biochim. Biophys. Acta-Biomembr. 1808, 29812994 (2011).

56. Taberner, F. J., Fernández-ballester, G., Fernándezcarvajal, A. \& Ferrer-montiel, A. Biochimica et Biophysica Acta TRP channels interaction with lipids and its implications in disease 2 . BBA - Biomembr. 1848, 1818-1827 (2015).

57. Liu, C. \& Montell, C. Forcing open TRP channels: Mechanical gating as a unifying activation mechanism. Biochem. Biophys. Res. Commun. 460, 22-25 (2015).

58. Devilliers, M. et al. Activation of TREK-1 by morphine results in analgesia without adverse side effects. Nat. Commun. 4, 2941 (2013).

59. Wan, Z. et al. PI(4,5)P2 determines the threshold of mechanical force-induced B cell activation. J. Cell Biol. jcb.201711055 (2018).

\section{MATERIALS AND METHODS}

\section{Expression and Purification of TREK-1.}

Expression and purification of TREK-1 was performed as previously explained ${ }^{44}$. Briefly, Pichia pastoris (SMD1163H) transformed with the TREK1 gene in pPICZ-B vector was grown in $2.8 \mathrm{~L}$ baffled flasks. Overnight cultures were grown overnight at $30 \mathrm{C}, 250 \mathrm{rpm}$ to an OD600 of $\sim 16$. Cells are then harvested and resuspended in minimal methanol doi:10.1083/jcb.201711055

60. Hill, R. Z. et al. The signaling lipid sphingosine 1phosphate regulates mechanical pain. Elife 7, e33285 (2018).

61. Brohawn, S. G., del Marmol, J., MacKinnon, R. \& del Mármol, J. Crystal Structure of the Human K2P TRAAK, a Lipid- and Mechano-Sensitive $\mathrm{K}+$ Ion Channel. Science (80-. ). 335, 436-441 (2012).

62. Su, Z., Brown, E. C., Wang, W. \& Mackinnon, R. Novel cell-free high-throughput screening method for pharmacological tools targeting $\mathrm{K}+$ channels. Proc. Natl. Acad. Sci. U. S. A. 113, 1602815113(2016).

63. Tanaka, K. A. K. et al. Membrane molecules mobile even after chemical fixation. Nat. Methods 7, 865866 (2010).

64. Raghunathan, K. \& Kenworthy, A. K. Dynamic pattern generation in cell membranes: Current insights into membrane organization. Biochim. Biophys. acta. Biomembr. 1860, 2018-2031 (2018).

65. Wang, H., Feng, Z., Del Signore, S. J., Rodal, A. A. \& $\mathrm{Xu}, \mathrm{B}$. Active Probes for Imaging Membrane Dynamics of Live Cells with High Spatial and Temporal Resolution over Extended Time Scales and Areas. J. Am. Chem. Soc. 140, 3505-3509 (2018).

66. Drago, I. \& Davis, R. L. Inhibiting the Mitochondrial Calcium Uniporter during Development Impairs Memory in Adult Drosophila. Cell Rep. 16, 27632776 (2016).

67. Lin, B.-J. et al. Lipid rafts sense and direct electric field-induced migration. Proc. Natl. Acad. Sci. U. S. A. 114, 8568-8573 (2017).

68. Coltharp, C., Yang, X. \& Xiao, J. Quantitative analysis of single-molecule superresolution images. Curr. Opin. Struct. Biol. 28, 112-121 (2014).

media and incubation temp is reduced to $25 \mathrm{C}$. Induction was maintained by the addition of $0.5 \%$ methanol every 12 hours. Expression was continued for $\sim 48-60$ hours. Cells were pelleted, frozen in N2I, and stored at $-80 \mathrm{C}$.

Cells were milled and powder was added to lysis buffer $(50 \mathrm{mM}$ Tris $\mathrm{pH} 8.0,150 \mathrm{mM} \mathrm{KCl}, 60 \mathrm{mM}$ dodecyl- $\beta$-D-maltoside (DDM), $0.1 \mathrm{mg} / \mathrm{mL}$ DNAse $1,0.1 \mu \mathrm{g} / \mathrm{ml}$ pepstatin, $1 \mu \mathrm{g} / \mathrm{ml}$ leupeptin, $1 \mu \mathrm{g} / \mathrm{ml}$ 
aprotinin, $0.1 \mathrm{mg} / \mathrm{ml}$ soy trypsin inhibitor, $1 \mathrm{mM}$ benzamidine, and $0.1 \mathrm{mg} / \mathrm{ml} \mathrm{AEBSF}$, with $1 \mathrm{mM}$ phenylmethylsulfonyl fluoride added immediately before use) at a ratio of $1 \mathrm{~g}$ pellet $/ 4 \mathrm{~mL}$ lysis buffer. After stirring for 4 hours membranes were extracted at 35,000 $\mathrm{xg}$ for $45 \mathrm{~min}$ and supernatant was applied to cobalt resin (Clontech) column by gravity flow. Column was serially washed and eluted in IMAC buffer(50 mM Tris pH 8.0, $150 \mathrm{mM}$ $\mathrm{KCl}, 4 \mathrm{mM} \mathrm{DDM}, \mathrm{pH}$ 8) with $30 \mathrm{mM}$ and $300 \mathrm{mM}$ imidazole respectively. Eluted protein was concentrated and applied to Superdex 200 column (GE Healthcare) equilibrated in buffer (20 mM Tris $\mathrm{pH}$ 8.0, 150 mM KCl, 1 mM EDTA, 2 mM DDM).

Flux Assay

The flux assay was performed similarly as previously published ${ }^{61}$. Briefly, $5 \mu \mathrm{L}$ of sonicated proteoliposomes was added to $195 \mu \mathrm{L}$ of flux assay buffer $(150 \mathrm{mM} \mathrm{NaCl}, 20 \mathrm{mM}$ HEPES pH 7.4, $2 \mu \mathrm{M}$ ACMA) in duplicates in a black 96-well plate (Costar 3915). A protocol was set up on a Tecan M200 Pro to initially read the fluorescence (excitation 410, emission 490) every twenty seconds for one minute as a baseline. The temperature inside the plate reader was set at $25^{\circ} \mathrm{C}$. Then, using the protonophore CCCP $(1 \mu \mathrm{M}$ final concentration), we collapsed the electrical potential allowing protons into the vesicles. Fluorescence was read every twenty seconds for seven minutes. Next, the potassium-selective ionophore valinomycin (20nM final concentration) was added to terminate the chemical gradient, and fluorescence was read every twenty seconds for five to ten minutes. An average of the duplicates was taken, and then the data was normalized similar to that published in Su et al ${ }^{62}$. Briefly, at each time point $\left(F-F_{\text {start }}\right) /\left(F_{\text {start }}-F_{\text {end }}\right)$ was calculated, where $F$ is the fluorescent value at that time, $F_{\text {start }}$ is the average of the first four initial readings before the addition of CCCP, and $F_{\text {end }}$ is the final fluorescent value after 5 minutes of valinomycin. Next, control proteoliposome flux was normalized to 1 throughout CCCP, and the TREK flux was normalized to the control accordingly. In flux assay figures, addition of CCCP occurs at $t=80$ seconds and ends at $t=520$ seconds before valinomycin was added.

Cell Culture and Gene Expression

HEK293t cells (ATCC Cat\# CRL-3216, RRID:CVCL_0063) were maintained in the solution consisting of the DMEM (Corning cellgro) culture media, 10\% FBS, 100 units $/ \mathrm{mL}$ penicillin, and 100 $\mu \mathrm{g} / \mathrm{mL}$ streptomycin. Cells were plated on poly-Dlysine-coated $12 \mathrm{~mm}$ microscope cover glass $\sim 12 \mathrm{~h}, \sim 36 \mathrm{~h}$, or $\sim 60 \mathrm{~h}$ before transfection in low confluence $(5 \%, 2.5 \%$, or $1.25 \%)$. Genes for target proteins were transiently co-transfected to HEK293t cells with X-tremeGENE 9 DNA transfection agent (Roche Diagnostics). Full-length human TREK-1(hTREK-1) with C-terminus GFP tag in $\mathrm{pCEH}$ vector was a gift from Dr. Stephen Long. Mouse PLD2 constructs(mPLD2) without GFP tag in pCGN vector were gifts from Dr. Michael Frohman. Both functional (mPLD2) and inactive mutant (mPLD2-K758R, single mutation) form of mPLD2 were used together blindly to test mPLD2 effect on hTREK-1. hTREK-1 was cotransfected with mPLD2 or K758R with the $1(0.5 \mathrm{~g}$ of hTREK-1):4(2g of PLD2) ratio ${ }^{35}$. All the salts for internal/external solutions were purchased from either Sigma or Fisher Scientific.

\section{Electrophysiology}

The transfected HEK293t cells were used in 24 36 hr. after transfection. Standard excised inside-out patch-clamp recording procedure for TREK-1 was performed following the lead of others (Brohawn et al., 2014; Honore et al, 2006; Coste et al., 2010). Currents were recorded at room temperature with Axopatch 200B amplifier and Digidata 1440A (Molecular Devices). Borosilicate glass electrode pipettes (B150-86-10, Sutter Instrument) were pulled with the Flaming/Brown micropipette puller (Model P-1000, Sutter instrument) resulting in 3 6 $\mathrm{M} \Omega$ resistances with the pipette solution (in $\mathrm{mM}$ ): $140 \mathrm{NaCl}, 5 \mathrm{KCl}, 1 \mathrm{CaCl}$, $3 \mathrm{MgCl} 2,10 \mathrm{TEA}-\mathrm{Cl}, 10$ HEPES, pH 7.4 (adjusted with $\mathrm{NaOH}$ ). Bath solution consists of (in $\mathrm{mM}$ ): $140 \mathrm{KCl}, 3 \mathrm{MgCl} 2,5$ EGTA, 10 TEA-Cl, 10 HEPES, pH 7.2 (adjusted with $\mathrm{KOH})$. Low concentration of TEA $(10 \mathrm{mM})$, which has been known to be insensitive to TREK1 current (Piechotta et al., 2011), was added into both pipette/bath solutions to block the endogenous potassium channels in HEK293 cells (Thomas and Smart, 2005). Patch electrodes were wrapped with parafilm to reduce capacitance. Currents measured using Clampex 10.3(Molecular Devices) were filtered at $1 \mathrm{kHz}$, sampled at $20 \mathrm{kHz}$, and stored on a hard disk for later analysis. Pressure clamping on the patch was performed using high speed pressure clamping system (ALA Scientific) through the Clampex control. Data was 
analyzed off-line by a homemade procedure using IgorPro 6.34A (WaveMetrics).

hTREK-1 current either co-expressed with MPLD2 or $\mathrm{K} 758 \mathrm{R}$ was activated by negative pressure steps from 60 to $0 \mathrm{mmHg}$ in $10 \mathrm{mmHg}$ decrements at $+30 \mathrm{mV}$ membrane potential, and 5 traces for each case were recorded and averaged for the analysis. Inside-out patch has generally less patch size variability than cell-attached recording when pressure clamped (Suchyna et al., 2009), but in other to further minimize the patch size variability in inside-out patches, patch size was estimated using a method described by Sakman and Neher (Sakman and Neher,1995), and the current density (I_density; $\mathrm{pA} / \mu^{2}{ }^{2}$ ) was calculated for the further analysis. Then, a Boltzman equation, I_density = base $+\{\max /[1+\exp ((\mathrm{P} 50-\mathrm{P}) /$ slope $)]\}$ was used to fit the data with a constraint of base $=1$ due to poor saturation of the current at high pressure. $P$ is the applied pressure, P50 is the pressure that activates $50 \%$ of maximum current, and slope shows the sensitivity of current activation by pressure. In some experiments with hTREK-1+K758R coexpression where the activated currents were too small to fit to the Boltzman equation, the current amplitude at $\mathrm{P}=-30 \mathrm{mmHg}$ (I_m30) was compared with its 5x standard deviation(I_5xSD). If I_m30 < I_5XSD, the experiment was excluded from the Boltzman equation fitting and corresponding P50slope analysis. This empirical rule (we call it $5 \times S D$ rule) can discern 4 out of 5 wilt type cell-attached recording cases as null experiments suggesting that it could be a usable/useful empirical criterion for our experiment. Then, the current density at -60 $\mathrm{mmHg}$ and P50-slope data were used for statistical analysis. Mann Whitney test was done to assess statistical significance using Prism6 (GraphPad software), and outliers were eliminated using a built-in function in Prism with $Q=1 \%$. The values represented are Mean +/- SEM.

Pressure clamp instrument delay was calculated as follows: the pressure device requires $\sim 4 \mathrm{~ms}$ as measured within the device itself. The distance the pressure must travel from the device to the pipette tip is $\sim 100 \mathrm{~cm}$ at a propagation speed of 34,300 $\mathrm{cm} / \mathrm{s}$ (the speed of sound in air). This results in a delay of $\sim 2.9 \mathrm{~ms}$ for propagation to the patch. The distance and speed of pressure propagation through the pipette tip and solution was considered negligible. The resulting current was compared to a baseline current of $1 \mathrm{~ms}$ before pressure clamp began. To determine the first significant time point, a $1 \mathrm{~ms}$ running average was taken of the postclamped current and compared to baseline using a one-way ANOVA followed by the Fisher's LSD test. Cell Culture

All cells were grown in Dulbecco's Modified Eagle Medium (DMEM) containing 10\% fetal bovine serum (FBS) and $1 \%$ penicillin/streptomycin unless otherwise noted. C2C12 cells (ATCC Cat\# CRL1772, RRID:CVCL_0188) were changed to a serum-free media containing no FBS or antibiotics 24 hours prior to experimentation unless otherwise noted. For the in vivo assay, PBS-glucose buffer contained D-glucose $(20 \mathrm{mM})$ in PBS (VWR, 45000-446).

Fixed cell preparation

C2C12 cells were grown to $80 \%$ confluence and then allowed to differentiate overnight in serum free media. Cells were rinsed, treated as needed, and then fixed with $3 \%$ paraformaldehyde and $0.1 \%$ glutaraldehyde for $10 \mathrm{~min}$ to fix both protein and lipids. Glutaraldehyde was reduced with $0.1 \%$ $\mathrm{NaBH} 4$ for 7 min followed by three-10 min washes with PBS. Cells were permeabilized for 15 min with $0.2 \%$ Triton $\mathrm{X}-100$, blocked with $10 \%$ BSA $/ 0.05 \%$ Triton/PBS at rt. for $90 \mathrm{~min}$. Primary antibody (PLD2, Cell Signalling \#13891; TREK-1, Santa Cruz \#sc-50412; PIP2, Echelon Biosciences \#zP045) was added to a solution of $5 \%$ BSA $/ 0.05 \%$ Triton/PBS for $60 \mathrm{~min}$ at $\mathrm{rt}$ at a concentration of $1: 100$ followed by 5 washes with $1 \%$ BSA $/ 0.05 \%$ Triton/PBS for 15 min each. Secondary antibody (Life Technologies \#A21244 and A21235; cy3B antibodies were produced as described previously ${ }^{5}$ ) was added in the same buffer as primary for $30 \mathrm{~min}$ at $\mathrm{rt}$ followed by 5 washes as above. A single 5 min wash with PBS was followed by a post-fix with fixing mixture, as above, for 10 min w/o shaking. This was followed by three- $5 \mathrm{~min}$. washes with PBS and two-3 min. washes with $\mathrm{dH} 2 \mathrm{O}$. Cells only receiving $\mathrm{CT} \times \mathrm{B}$ treatment were not permeabilized.

The dual-fixation protocol is used to minimize any effects from post-fixation aberrations. While always good practice for super-resolution in general, this dual-fixation also ensures that the movement of any molecule of interest which may not have been immobilized by the initial fixation can be fully immobilized after labeling since the antibodies or toxins used for labeling will be efficiently crosslinked during this post-labeling fixation step. While 
some have proposed that this problem should be solved by adding the label before the initial fixation $^{63}$ we believe that in the absence of easilyattainable monomeric labeling molecules it would have likely led to clustering artifacts due to the (often) multimer nature of the labeling proteins.

Shear force was applied to cells in ibidi $\mu$-Slide $\mathrm{I}^{0.4}$ Luer chambers with a flow rate calibrated to apply 3.0 dynes $/ \mathrm{cm}^{2}$. Fixation media (see above) was applied to cells using a syringe pump (Harvard Apparatus PHD ULTRA) and kept at $37^{\circ} \mathrm{C}$ using an in-line heater (Warner SH-27B).

\section{Imaging Protocols}

Super-resolution dSTORM imaging

Images were recorded with a Vutara 352 superresolution microscope (Bruker Nano Surfaces, Salt Lake City, UT) which is based on the 3D Biplane approach38. Super-resolution images were captured using a Hamamatsu ORCA Flash4.0 sCMOS camera and a $60 x$ water objective with numerical aperture 1.2. Data were analyzed by the Vutara SRX software (version 5.21.13). Single molecules were identified by their brightness frame by frame after removing the background. Identified particles were then localized in three dimensions by fitting the raw data in a customizable region of interest (typically $16 \times 16$ pixels) centered on each particle in each plane with a 3D model function that was obtained from recorded bead data sets. Fit results were stored as data lists for further analysis. Fixed samples were imaged using a $647 \mathrm{~nm}$ and $561 \mathrm{~nm}$ excitation lasers, respectively, and $405 \mathrm{~nm}$ activation laser in photoswitching buffer comprising of $20 \mathrm{mM}$ cysteamine (Sigma, \#30070), 1\% betamercaptoethanol (Sigma, \#63689) and oxygen scavengers (glucose oxidase (Sigma \#G2133) and catalase (Sigma \#C40)) in 50mM Tris (Affymetrix, \#22638100)+10 mM NaCl (Sigma, \#S7653) +10\% glucose (Sigma, \#G8270) at pH 8.0 at $50 \mathrm{~Hz}$ and maximal powers of $647 \mathrm{~nm}, 561 \mathrm{~nm}$ and 405 lasers set to 8,10 , and $0.05 \mathrm{~kW} \mathrm{cm-2} \mathrm{respectively.} \mathrm{Live}$ cell imaging was performed in DMEM supplemented with oxygen scavengers and $0.1 \%$ betamercaptoethanol in $50 \mathrm{mM}$ Tris $+10 \mathrm{mM}$ buffer $+2 \%$ glucose. An autocorrelative algorithm ${ }^{38}$ was used to correct for drift correction.

Pair correlation and cluster analysis was performed using the Statistical Analysis package in the Vutara SRX software. Pair correlation analysis is a statistical method used to determine the strength of correlation between two objects by counting the number of points of probe 2 within a certain donut-radius of each point of probe 1 . This allows for localization to be determined without overlapping pixels as done in traditional diffractionlimited microscopy. Localization at super resolution is beyond techniques appropriate for diffractionlimited microscopy such as Pearson's correlation coefficient.

Fluorescence Recovery After Photobleaching (FRAP)

For fixation studies $\mathrm{N} 2 \mathrm{a}$ and $\mathrm{C} 2 \mathrm{C} 12$ cells were grown in DMEM with 10\% FBS until 16 hours before use in which they were switched into serum free DMEM. On the day of the experiment, DMEM in live cells was replaced with DMEM w/o phenol red. Fixed cells were rinsed once with PBS and then put into a mixture of PBS with $3 \%$ PFA and $0.1 \%$ glutaraldehyde for $20 \mathrm{~min}$ at $37 \mathrm{C}$. Fixed cells were then rinsed with PBS $5 \times 5$ min and then placed back into phenol-free DMEM. CTxB (ThermoFisher C34778, $100 \mathrm{ug} / \mathrm{ml}$ ) was then applied 1:200 into each plate and allowed to incubate for $>30$ min before imaging. Imaging and data collection was performed on a Leica SP8 confocal microscope with the Application Suite $X$ v.1.1.0.12420. 5 images were taken as baseline after which a selection of 1 or more ROI were bleached at $100 \%$ laser power for 6-8 frames. Recovery was measured out to $5 \mathrm{~min}$ and fluorescence of the $\mathrm{ROI}(\mathrm{s})$ were quantified. The fluorescence before bleaching was normalized to 1 and after the bleaching step was normalized to 0 .

Drosophila Assays

For behavior experiments, 1 to 5-day old flies were collected in vials containing $\sim 50$ flies at least 12 hours before the experiment. Flies were allowed to acclimate to behavior room conditions for $>30 \mathrm{~min}$ (dim red light, $\sim 75 \%$ humidity) before each assay. Shock avoidance was tested by placing flies in a Tmaze where they could choose between an arm shocking at the indicated voltage every 2 seconds and an arm without shock. Flies were given 2 min to choose which arm, after which flies were collected and counted to determine the shock avoidance index for each voltage and genotype. Control and knockout flies were alternated to avoid any preference and the arm used for shock was also alternated to control for any non-shock preference in the T-maze itself.

Arousal threshold protocol has been described in detail previously ${ }^{48}$. Briefly, animals were exposed 
hourly to a series of vibrations of increasing intensity ranging from 0.8 to $3.2 \mathrm{~g}$, in steps of 0.6 g. Stimuli trains were composed of $200 \mathrm{~ms}$ vibration with $800 \mathrm{~ms}$ inter-vibration interval and 15 $\mathrm{s}$ inter-stimuli train interval. Stimulation intensity and timing were controlled using pulse-width modulation via an Arduino UNO and shaft-less vibrating motors (Precision Microdrives, model 312-110). Arousal to a given stimulus was assigned when an animal (1) was inactive at the time of the stimulus, (2) satisfied a given inactivity criteria at the time of the stimulus, and (3) moved within the inter-stimuli train period (15 s) of that stimulus.

Statistics

All statistical calculations were performed using a Student's t-test unless otherwise noted. Significance is noted as follows: ns: $p>0.05$; ${ }^{*}: p<0.05 ;{ }^{* *}: p<0.01 ;{ }^{* * *}: p<0.001$. 


\section{Phospholipase D Transduces Force to TREK-1 Channels in a Biological Membrane}

E. Nicholas Petersen ${ }^{1,4}$, Manasa Gudheti ${ }^{5}$, Mahmud Arif Pavel ${ }^{1}$, Keith R. Murphy ${ }^{2,3}$, William W. Ja ${ }^{2,3}$, Erik M. Jorgensen ${ }^{5}$, Scott B. Hansen ${ }^{1 *}$ 


\section{SUPPLEMENTAL FIGURES}

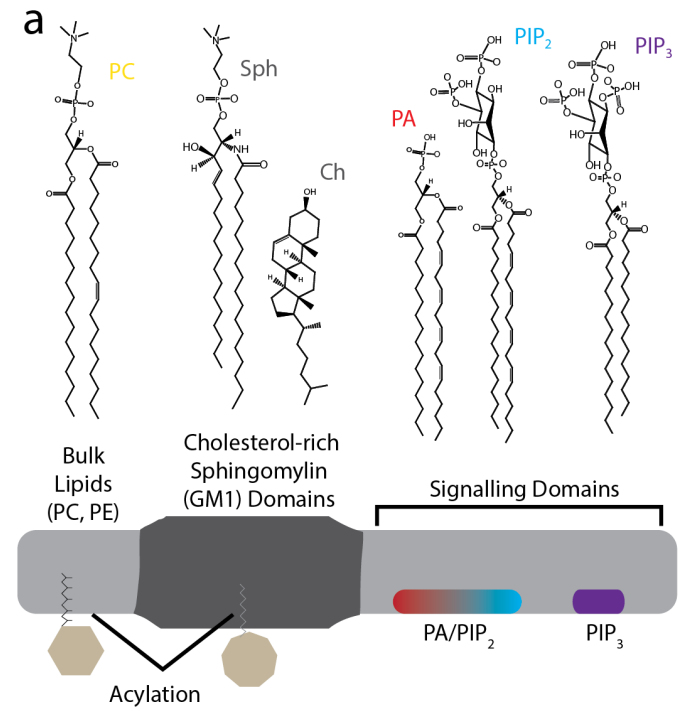

b Domain-Targeting Acylations

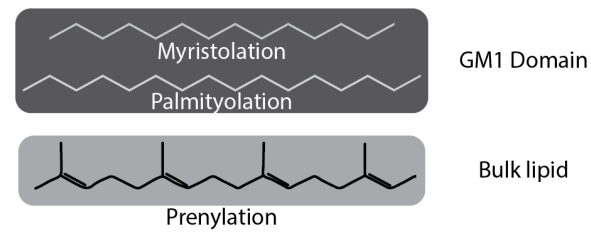

C
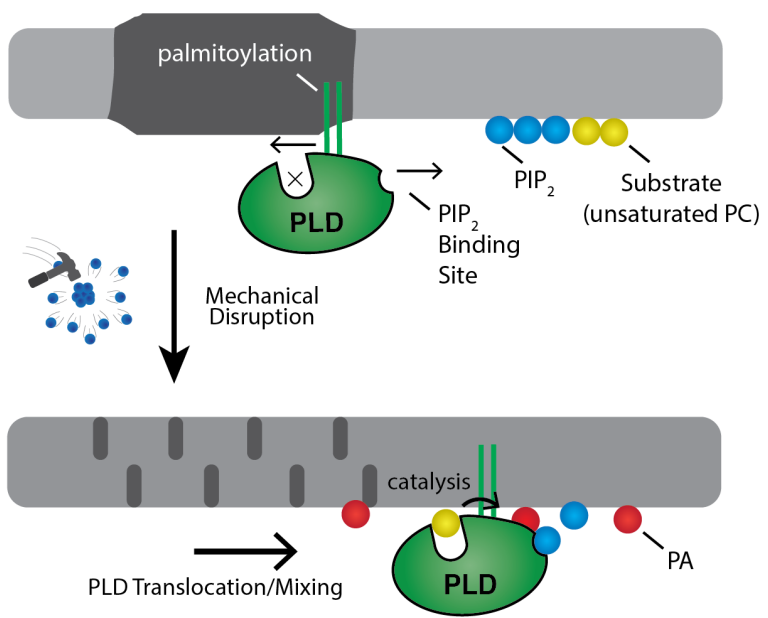

d

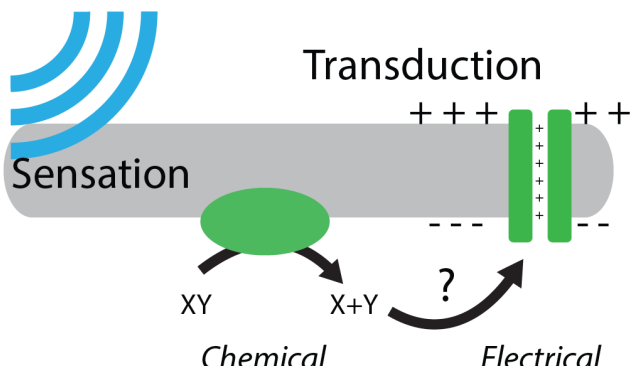

Supplemental Figure 1. Role of lipids and lipid order in mechano-transduction.

The plasma membrane is composed of lipids which are able to cluster into separate and distinct domains with unique properties such as thickness and charge (a). These domains contain proteins which localize to them through post-translational acylation (b). Domain localization exposes proteins to micro-environments which can regulate their activity. For example, our previous findings showed that PLD is localized to GM1-labeled domains due to its palmitoylation sites (c, top). This sequesters PLD from high concentrations of substrate (phosphatidylcholine) and activating lipid $\left(\mathrm{PIP}_{2}\right)$. Upon chemical or mechanical disruption of the domain, the environment changes, instead favoring PLD translocation and activation (b, bottom) by substrate presentation. This process is a form of cellular transduction. (d) Transduction is known to occur directly between a mechanical to chemical signal through the use of raft-localized enzymes, but it is unknown whether electrical transduction also occurs directly or can be facilitated through a secondary mechanism. 
a

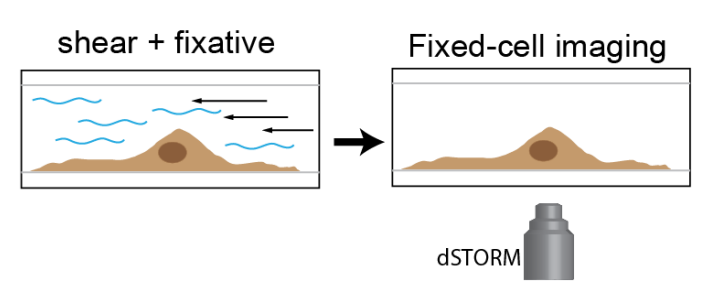

b

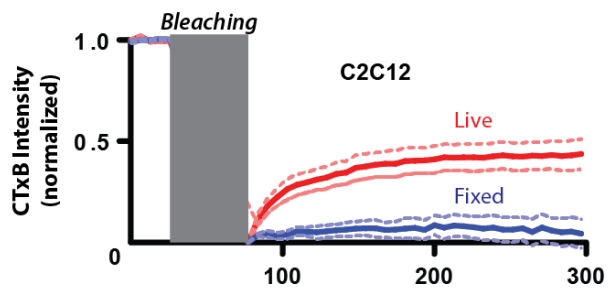

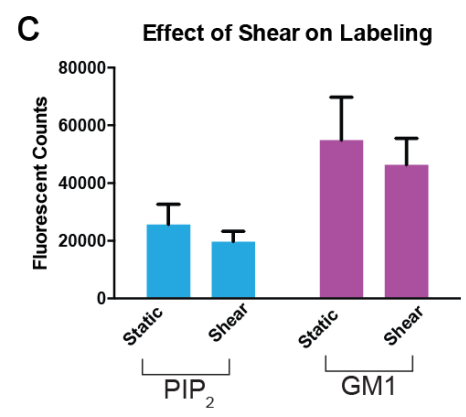

Supplemental Figure 2. Observing cellular changes in response to mechanical stimulation.

(a) In order to image the cells in a sheared state, cells were plated in a parallel plate chamber and fixed while shearing. After shearing, cells were fixed, stained, and imaged identically to static samples with dSTORM. (b) Recent research has shown variability in the detection of domains in cells based on technique and labeling molecules ${ }^{29,64,65}$. Because of its pentavalent nature, CTXB has been suggested to induce lipid rafts post-fixation due to the idea that lipids remain mobile after fixation. Since single particle tracking (SPT) has shown that some molecules can remain mobile after fixation on a nanoscale $^{63}$, we wanted to determine if larger scale diffusion (which could induce artificial domains) existed after chemical fixation. Fluorescence recovery after photobleaching (FRAP) imaging shows that PFA and glutaraldehyde fixation is sufficient to restrict the large-scale movement of lipids in fixed cells when compared to live cells. So while nano-scale grouping due to CTxB application may occur, it does not affect our mechanism which relies on intra-domain transport. (c) For both $\mathrm{PIP}_{2}$ and GM1 labeling, shear did not decrease the overall counts with dSTORM significantly ( $p>0.05$, Student's t-test). The slight decrease noticed could be due to internalization of labeled targets or simply to cell-to-cell variation. 
a

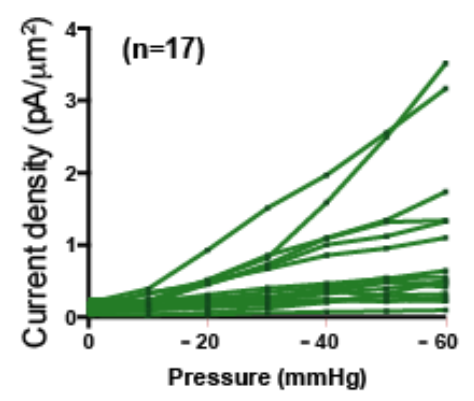

b

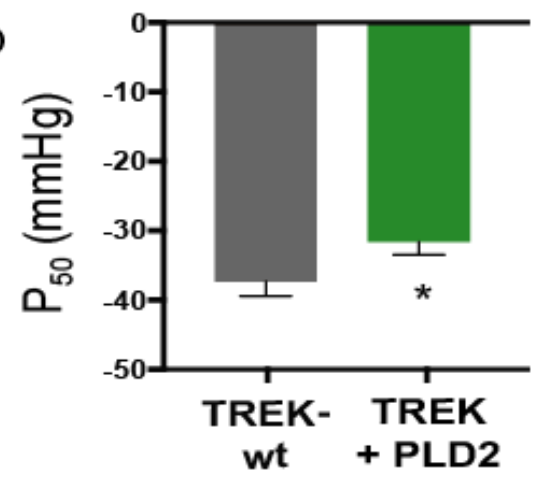

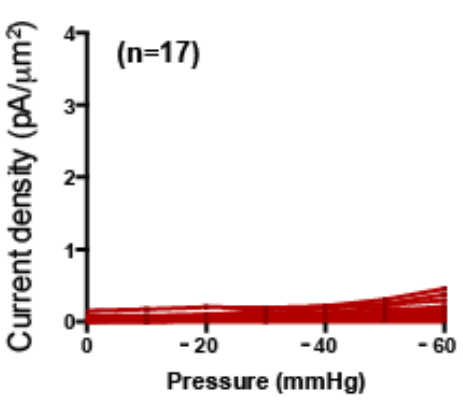

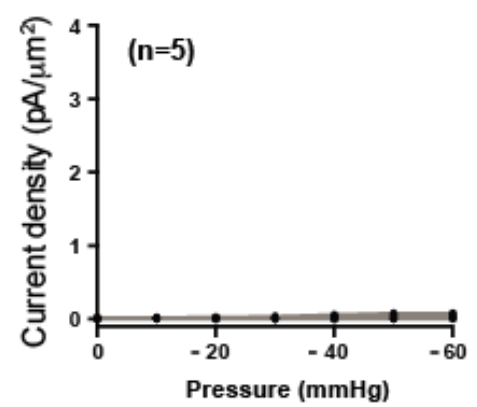

C

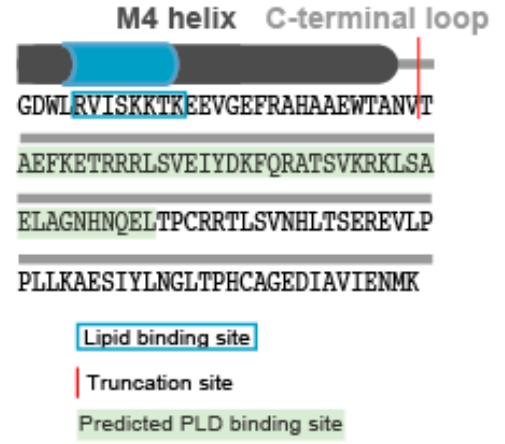

Supplemental Figure 3. Electrophysiology details and methods.

(a) Individual cell traces for TREK-1+PLD2 (green), TREK-1+xPLD2 (red), and TREK-1-truncated (grey). (b) The pressure required to open TREK is decreased when PLD is overexpressed $(p<0.05$, $\mathrm{n}=15-20$ ), confirming that PLD has a role in the mechanical activation of TREK. (c) Diagram of the Cterminal end of TREK-1 showing the truncation site (red) used and the predicted PLD binding site for PLD2. The last transmembrane helix (M4) is shown as a grey cylinder, and the anionic lipid binding site is highlighted in blue. 

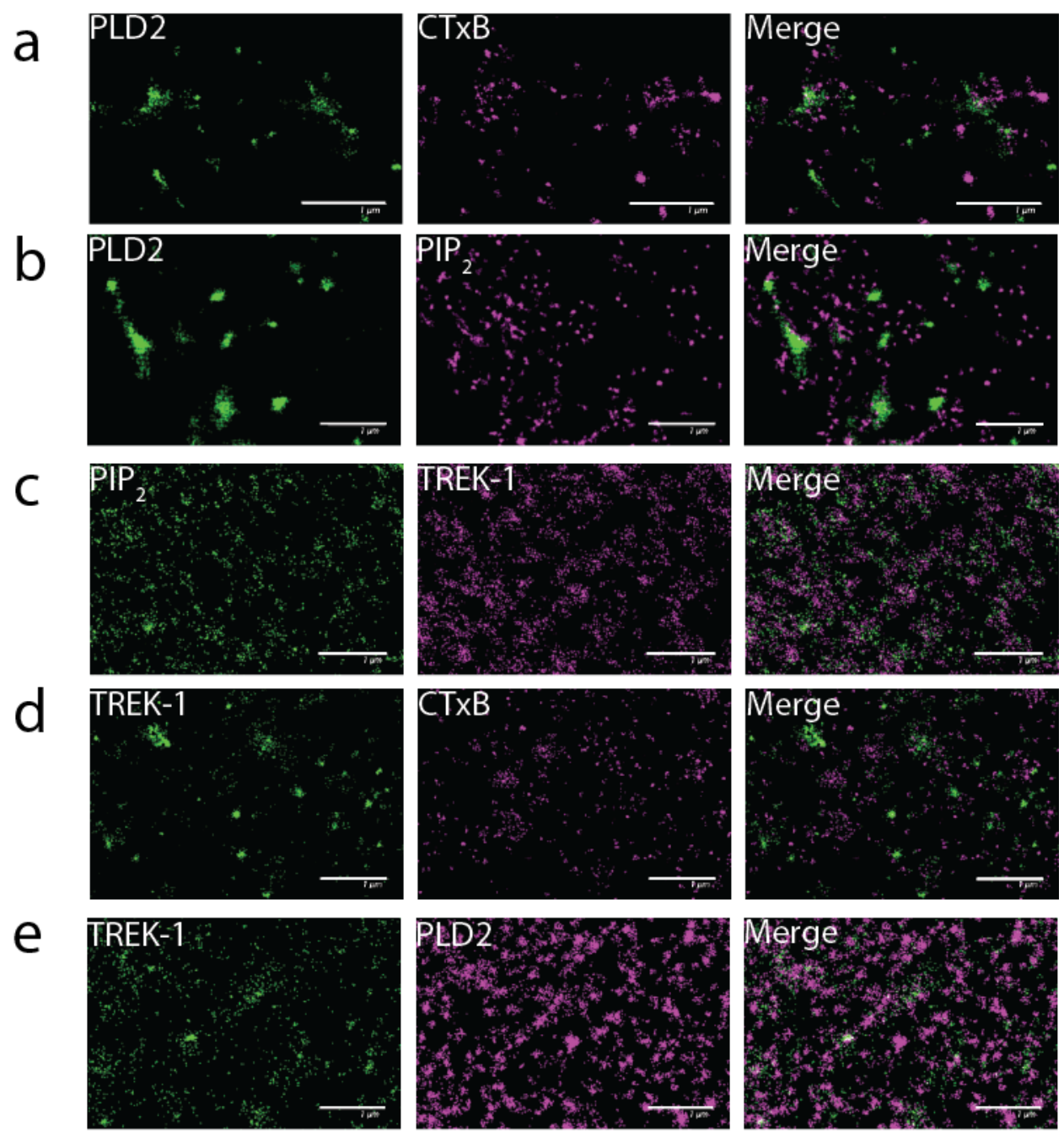

$f$
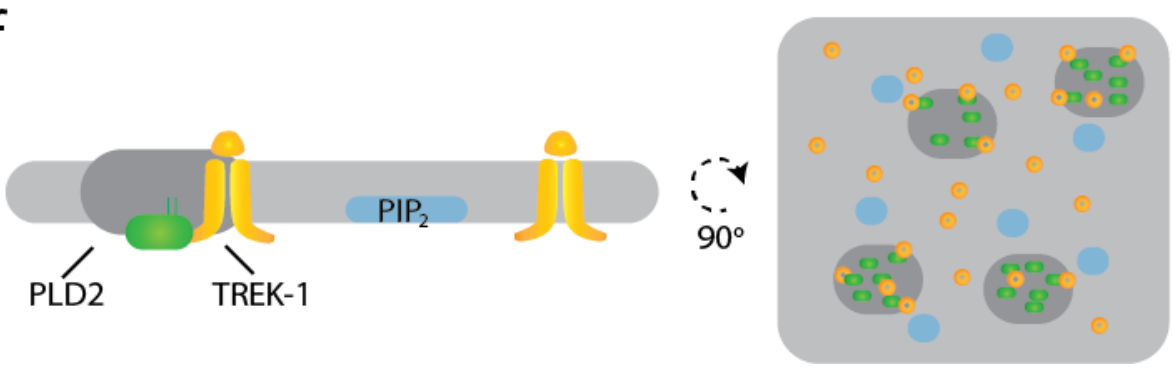

Supplemental Figure 4. Cellular organization of PLD2 and TREK-1 in rafts before shear. (a-e) dSTORM imaging of membrane proteins and lipids show how membrane components are organized. As expected, PLD2 and CTxB are typically found in clusters near one another (a) and are excluded from $\mathrm{PI}(4,5) \mathrm{P}_{2}$ domains (b). In contrast, TREK-1 does not seem to cluster strongly in with either $\mathrm{PI}(4,5) \mathrm{P}_{2}$ (c), CTxB (d), or PLD2 (e). In (f) is shown a scenario in which this lack of preference could be observed. Since PLD2 and TREK-1 are known binding partners, some TREK-1 could localize with PLD2 in microdomains, while the remaining TREK protein would be found in the bulk membrane. 
a
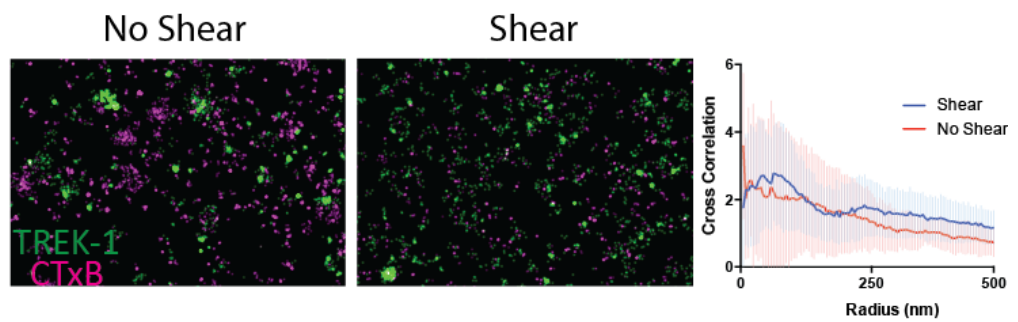

b
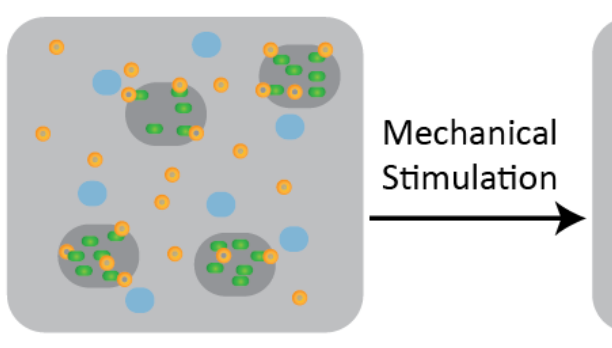

$\mathrm{C}$
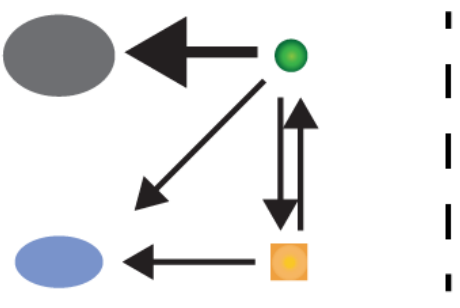

[-TREK-1

- - PLD2

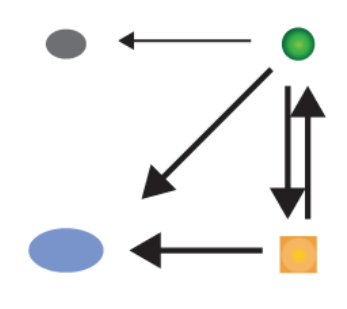

Supplemental Figure 5. Shear localization changes of TREK-1 and associated lipids.

(a) Shear is shown not to affect the correlation between TREK-1 and lipid domains labeled by CTxB. This is likely because TREK-1 does not strongly associate with these domains before shear, and CTxB has an almost random distribution afterward, making it unlikely that anything would localize strongly (see b). (c) Force diagram showing how GM1 domain disruption changes the predominant attractive force for associated proteins. This is one explanation for why translocation could occur under these conditions. 
a

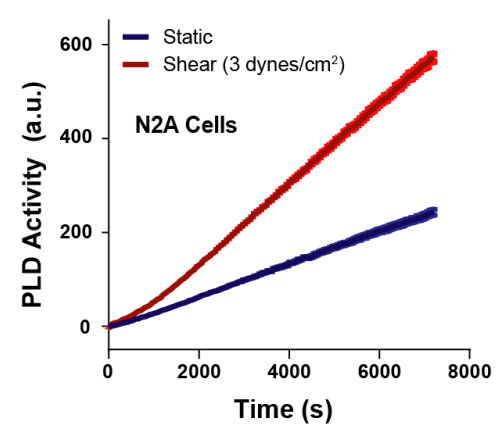

$\mathrm{b}$
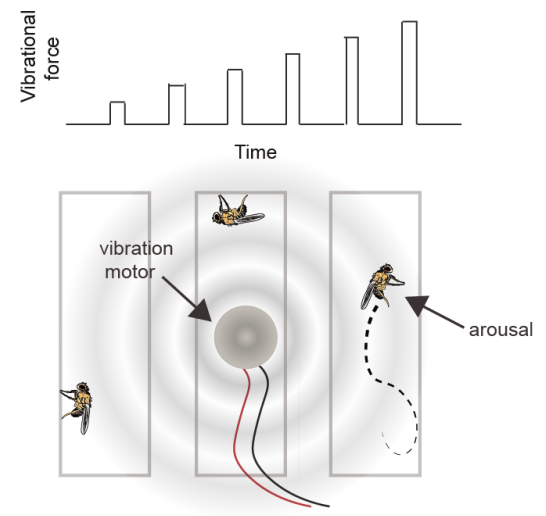

C

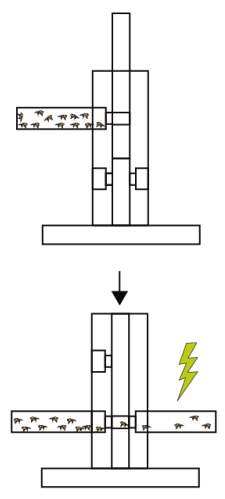

d

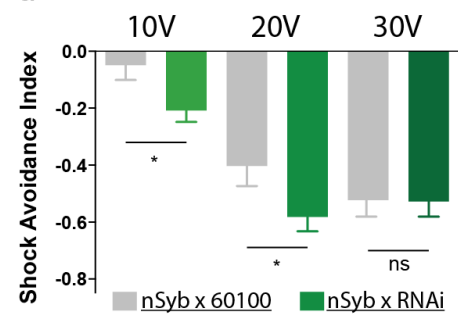

e

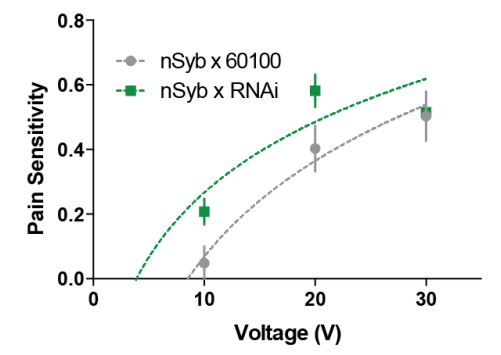

Supplemental Figure 6. Pan-neuronal knockdown of pld in Drosophila results in a change in sensitivity to shock.

(a) PLD in N2a cells show an increase in activity when subjected to mechanical force. (b) Mechanical stimulation was applied in a series of six increasing vibrations (top). Vibration motor was attached to the back of the chamber with the flies and flies were monitored using a web camera for stimulation-induced arousal (bottom). (c) Shock avoidance responses were measured by placing the PLD-KD flies at the choice-point of a T maze in which they could then choose between a chamber inducing noxious shock or a non-shock control ${ }^{66}$. Flies were subjected to increasing voltages of shock and an avoidance ratio was measured as previously described ${ }^{66}$. Shock avoidance index (Al) was calculated as $A I=$

(\# flies shock arm - \# flies control arm)/\# flies total. PLD-KD flies showed an increased sensitivity to shock when compared with wild-type flies. (d) PLD ${ }^{\text {RNAi }}$ flies had a higher aversion to shock at $10 \mathrm{~V}$ $(p=0.0213, n=21)$ and 20V $(p=0.0492, n=27-30)$, but not at 30V $(p=0.672, N=12)(e)$ Observing the inverse of this metric we obtain a pain sensitivity curve in which we observe a right-shift when the pld gene was knocked down (Fig. 3d). In addition to direct activation of nerves by voltage, electric current were recently shown to mechanically perturb domain clustering ${ }^{67}$ which could contribute to the change in pain threshold we observed in flies. 

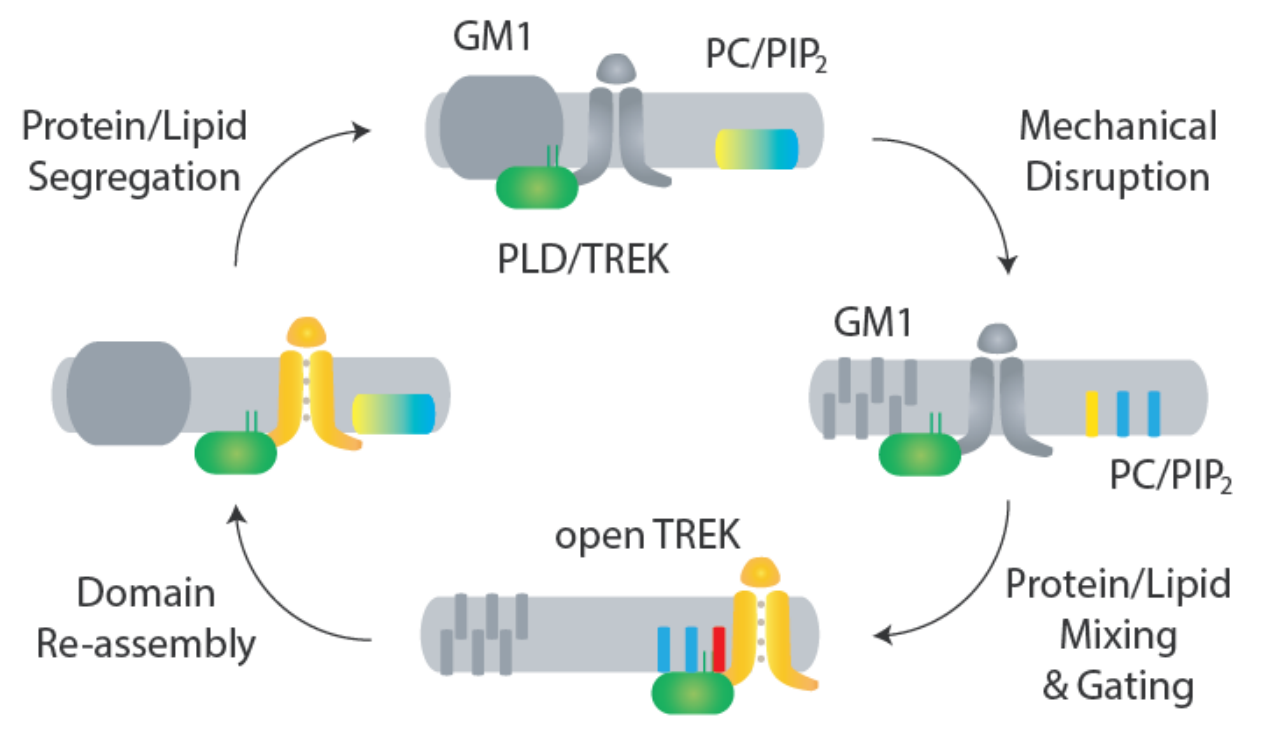

$\mathrm{PA} / \mathrm{PIP}_{2}$

Supplemental Figure 8. Proposed Lipid mixing model of TREK-1 mechanosensation.

In domain-mediated mechanosensation, in an unstimulated state, domains are intact, isolating the PLD2/TREK-1 complex from their respective activating lipids (PC/PIP 2 , top). After stimulation, the raft is disrupted, causing the PLD2/TREK-1 complex to translocate (right) and eventually bind to PC and PIP2. Upon binding, PLD2 is activated (bottom) transducing the mechanical signal to a chemical signal which then binds to and activates TREK-1 in chemical-to-electrical transduction. Upon stimulation removal, the domain reforms (left) causing the complex to be re-sequestered, causing a return to steady-state conditions (top). 


\section{SUPPLEMENTAL DiscuSSION}

Curvature and hydrophobic mismatch are popular mechanisms to describe direct opening of ion channels. Unlike a channel, PLD2 is a soluble protein that associates with the membrane through palmitoylation. Hence, the PLD2 specific TREK-1 mechanosensitivity is unlikely through changes in bilayer thickness or membrane curvature. Rather bilayer thickness and membrane curvature are likely mechanisms reserved for regulation of TREK-1.

Also, great care was taken to ensure that conclusions presented here from the imaging were based not on single conditions, but on relative changes between two states. While there are likely conditions that cannot be fully accounted for, since all samples are treated identically (save for the treatment) any artifacts resulting from the protocol used will be largely negated, allowing for an accurate conclusion based on the comparison between the two states observed. Cross-correlation analysis already compensates for many of these variables such as changes in labeling density and overcounting ${ }^{68}$. These corrections apply both to the labeling protocol, as well as the size and correlation analysis which, while not claiming to be absolute, exhibit changes relative to one another. This is the data upon which our conclusions are based. It is worth mentioning that due to the closeness of the resolution ability of dSTORM with the reported sizes of the observed lipid domains that the estimates of relative size change are likely underestimated, increasing the significance for our conclusions on both size and protein association to these domains.

\section{SUPPLEMENTAL REFERENCES}

55. Tanaka, K. A. K. et al. Membrane molecules mobile even after chemical fixation. Nat. Methods 7, 865-866 (2010).

56. Coltharp, C., Yang, X. \& Xiao, J. Quantitative analysis of single-molecule superresolution images. Curr. Opin. Struct. Biol. 28, 112-121 (2014). 